PHYSICAL REVIEW B 87, 125422 (2013)

\title{
Scattering approach to frequency-dependent current noise in Fabry-Pérot graphene devices
}

\author{
Jan Hammer and Wolfgang Belzig \\ Fachbereich Physik, Universität Konstanz, 78457 Konstanz, Germany \\ (Received 5 March 2012; revised manuscript received 3 February 2013; published 20 March 2013)
}

\begin{abstract}
We study finite-frequency quantum noise and photon-assisted electron transport through a wide and ballistic graphene sheet between two metallic leads. The elementary excitations allow us to examine the differences between effects related to Fabry-Pérot-like interferences and signatures caused by correlations of coherently scattered particles in electron- and holelike parts of the Dirac spectrum. We identify different features in the current-current auto- and cross-correlation spectra and trace them back to the interference patterns of the product of transmission and reflection amplitudes, which define the integrands of the involved correlators. At positive frequencies, the correlator of the autoterminal noise spectrum with final and initial states associated to the measurement terminal is dominant. Phase jumps occur within the interference patterns of corresponding integrands, which also reveal the intrinsic energy scale of the two-terminal graphene setup. The excess noise spectra, as well as the cross-correlation ones, show large fluctuations between positive and negative values. Oscillatory signatures of the cross-correlation noise are due to an alternating behavior of the integrands.
\end{abstract}

DOI: 10.1103/PhysRevB.87.125422

PACS number(s): 72.70.+m, 72.10.-d, 73.21.La

\section{INTRODUCTION}

Ballistic electron transport ${ }^{1,2}$ in two-terminal graphene systems is in the focus of intensive studies ever since the pioneering experiments on single-layer carbon., ${ }^{3,4}$ The Dirac Hamiltonian $^{3,5}$ describes charge transport close to the chargeneutrality point and leads to a linear dispersion relation $\epsilon=$ $\hbar v_{F} k$. This allows to observe several relativistic phenomena in solid-state systems, such as Klein tunneling ${ }^{6-11}$ or the Zitterbewegung. ${ }^{12-14}$ In the very early works on graphene, the minimal conductivity ${ }^{12,15,16,18,19} G \approx e^{2} / h$ per valley and per pseudospin at the charge-neutrality point was found and stimulated the research on current and noise properties. The currentcurrent correlations around the minimal conductivity lead to a zero frequency sub-Poissonian Fano factor with a maximal value of $F=1 / 3,{ }^{20-24}$ remarkably similar to diffusive systems such as disordered metals. ${ }^{25-27}$ The suppression of the Fano factor below the Poissonian value originates from noiseless, open quantum channels that are found at all conductance minima in graphene-based two-terminal structures ${ }^{21}$ and can be explained as an interplay between Klein tunneling, resonant tunneling, and pseudospin matching. This pseudodiffusive behavior $^{28}$ is due to the special band structure of graphene. Without impurity scattering, coherent transport through such a graphene sheet ${ }^{29}$ gives rise to the same shot noise as in classical diffusive systems. The opening of a gap ${ }^{31}$ in the quasiparticle spectrum leads to an enhanced Fano factor. ${ }^{21}$ Such a gap can be opened, for example, in a Fabry-Pérot geometry ${ }^{32,33}$ or by photon-assisted tunneling, as shown recently for the case of a graphene $p$ - $n$ junction ${ }^{34}$ with a linear potential drop across the interface. ${ }^{36,37}$ There, Landau-Zener-like transitions stimulated by photon emission or absorption via resonant interaction of propagating quasiparticles in graphene with an irradiating electric field lead to hopping between different trajectories.

The scattering approach as put forward by Landauer and Büttiker ${ }^{38}$ has been applied to ac-driven charge transport ${ }^{39-42}$ through a metal-graphene interface with an abrupt potential change. ${ }^{43}$ The metal can be formed by a graphene lead strongly electrostatically doped by a gate potential, thus shifting the Dirac point far away from the Fermi energy. In this work, we adopt the formalism and parametrization introduced in Refs. 43 and 44 and calculate the finite-frequency current-current correlations at finite dc- and ac-bias voltages in the system depicted in Fig. 1. We complement recent results on ac transport in Fabry-Pérot graphene devices of Ref. 45, in which the influence of different boundary conditions, i.e., zigzag or armchair configurations, on the Fabry-Pérot patterns in a combined Tien-Gordon/tight-binding approach has been investigated. The influence on transmission properties of a time-dependent potential barrier in a graphene monolayer has been investigated. ${ }^{46}$ In our work, we use so-called infinite mass boundaries ${ }^{20,47}$ for the transverse direction. However, the different boundary conditions lead to indistinguishable results for short but wide $(L \ll W)$ graphene strips. $^{20}$

We focus on the interplay between the Dirac-spectrum with the Fabry-Perot interferences. This interplay results in a shot-noise spectrum that, despite some common basic features, strongly differs from the one that we discussed in a recent work ${ }^{48}$ on transport through a single resonant level. Interestingly, the well-known oscillations as a function of the electrochemical potential in graphene on a scale of the return frequency $\hbar v_{F} / L$, related to the length $L$ of the graphene sheet, can be seen as a reminiscence of Zitterbewegung. ${ }^{12}$ The role of the complex reflection amplitude and the onset of contributions of scattering states coming from terminal $\alpha$ and being scattered into terminal $\beta$ will be the key characteristics in our discussion of the results for the noise as a function of bias voltage and frequency. As a consequence of these onsets, the oscillations add up de- or constructively depending on the precise values of the voltage and frequency. In our setup, the separation of oscillations caused by the Fabry-Pérot reflections and effects caused by the band structure of the Dirac Hamiltonian is a priori not obvious. In both cases, phasecoherent transport is essential. However, for charge injection either into the conduction or the valence band only, effects like Zitterbewegung should not be present and all oscillating features of the noise spectra have to be of Fabry-Pérot nature. 


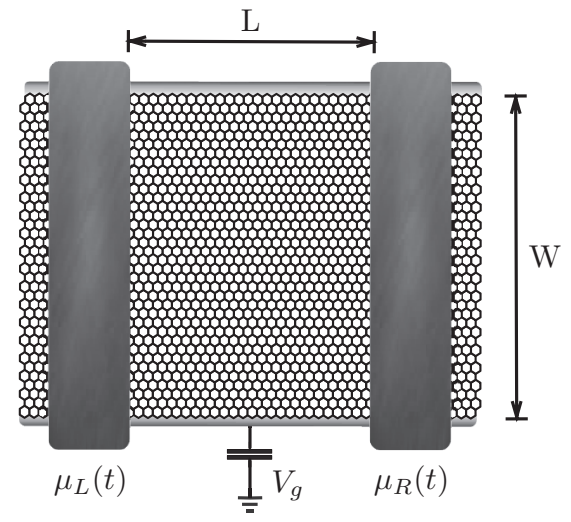

FIG. 1. Wide graphene strip $(W \gg L)$ between two heavily doped, metallic graphene leads. The Fermi level of the sheet can be tuned by modifying the electrochemical potential $V_{g}$ in the graphene sheet, i.e., via a gate voltage. Electron and hole states are injected via the time-dependent bias-voltages in left and right leads $\mu_{L / R}(t)$.

\section{DIRAC EQUATION AND SCATTERING FORMALISM}

The ballistic graphene ${ }^{49-51}$ sheet considered in the following can be described by the two-dimensional Dirac equation for the two-component spinor $\hat{\Psi}=\left(\hat{\Psi}_{1}, \hat{\Psi}_{2}\right)^{T}$ with indices referring to the two pseudospins of the carbon sublattices. Throughout this work, we will neglect intervalley scattering and Coulomb interactions. We only consider the interaction of the electrons with the radiation field in the form of photon-assisted transitions. With Fermi velocity $v_{F}$ the Dirac equation can be cast into the form

$$
\begin{aligned}
& {\left[-i v_{F} \hbar\left(\begin{array}{cc}
0 & \partial_{x}-i \partial_{y} \\
\partial_{x}+i \partial_{y} & 0
\end{array}\right)-\mu(\mathbf{x}, t)\right] \hat{\Psi}(\mathbf{x}, t)} \\
& =i \hbar \partial_{t} \hat{\Psi}(\mathbf{x}, t) \text {. }
\end{aligned}
$$

The electrochemical potential $\mu(\mathbf{x}, t)$ includes static and harmonically driven potentials in the leads plus a static electrochemical in the graphene sheet,

$$
\mu(\mathbf{x}, t)= \begin{cases}\mu_{L}+e V_{\mathrm{ac}, \mathrm{L}} \cos (\omega t), & \text { if } x<0, \\ e V_{g}, & \text { if } 0<x<L, \\ \mu_{R}+e V_{\mathrm{ac}, \mathrm{R}} \cos (\omega t), & \text { if } x>L .\end{cases}
$$

Making use of the Tien-Gordon ansatz, we write the solution to the time-dependent Dirac equation as a sum over photonassisted tunneling (PAT) modes:

$$
\begin{aligned}
\hat{\Psi}(\mathbf{x}, t) & =\hat{\Psi}_{0}(\mathbf{x}, t) e^{-i\left(e V_{\mathrm{ac}} / \hbar \omega\right) \sin (\omega t)} \\
& =\sum_{m=-\infty}^{\infty} J_{m}\left(\frac{e V_{\mathrm{ac}}}{\hbar \omega}\right) \hat{\Psi}_{0}(\mathbf{x}, t) e^{-i m \omega t}, \\
\text { where } & \hat{\Psi}_{0}(\mathbf{x}, t)=\hat{\Psi}_{0}(\mathbf{x}) e^{-i \epsilon t} .
\end{aligned}
$$

The advantage of this ansatz is that the scattering problem has to be solved for the time-independent case only. Therefore, in terminals $\gamma=L, R$, we define stationary solutions $\hat{\Psi}_{0}(\mathbf{x}, t)=$ $\hat{\Psi}_{\epsilon}(\mathbf{x}) e^{-i \epsilon t}$ by the equation

$$
\begin{aligned}
& {\left[\begin{array}{cc}
-i v_{F} \hbar\left(\begin{array}{cc}
0 & \partial_{x}-i \partial_{y} \\
\partial_{x}+i \partial_{y} & 0
\end{array}\right)-\mu_{\gamma}
\end{array}\right] \hat{\Psi}_{0}(\mathbf{x}) .} \\
& =\epsilon \hat{\Psi}_{0}(\mathbf{x}) .
\end{aligned}
$$

The basis states in graphene can be constructed as a superposition of left and right movers,

$$
\hat{\Psi}_{0}(\mathbf{x})=\sum_{k, q}\left[\Psi_{0,+}^{k, q}(\mathbf{x}) \hat{a}_{k, q}+\Psi_{0,-}^{k, q}(\mathbf{x}) \hat{a}_{-k, q}\right] .
$$

$\alpha_{q}(\epsilon)$ describes the angle between the momentum of a quasiparticle and its $y$-component $q$ in region $x=0 \ldots L$ of the graphene sheet. Then the pseudospinors can be parametrized as

$$
\begin{gathered}
\Psi_{0,+}^{k, q}(\mathbf{x})=\frac{e^{i q y+i k_{q}(\epsilon) x}}{\sqrt{\cos \alpha_{q}(\epsilon)}}\left(\begin{array}{c}
e^{-i \alpha_{q}(\epsilon) / 2} \\
e^{i \alpha_{q}(\epsilon) / 2}
\end{array}\right), \\
\Psi_{0,-}^{k, q}(\mathbf{x})=\frac{e^{i q y-i k_{q}(\epsilon) x}}{\sqrt{\cos \alpha_{q}(\epsilon)}}\left(\begin{array}{c}
e^{i \alpha_{q}(\epsilon) / 2} \\
-e^{-i \alpha_{q}(\epsilon) / 2}
\end{array}\right) .
\end{gathered}
$$

Here, the dispersion is given by $\epsilon=\hbar v_{F} \sqrt{q^{2}+k^{2}}$. The wave vector $k_{q}(\epsilon)$ and the angle $\alpha_{q}(\epsilon)$ are defined as

$$
\begin{aligned}
& \alpha_{q}(\epsilon)=\arcsin \left(\frac{\hbar v_{F} q}{\epsilon+e V_{g}}\right), \\
& k_{q}(\epsilon)=\frac{\epsilon+e V_{g}}{\hbar v_{F}} \cos \left[\alpha_{q}(\epsilon)\right] .
\end{aligned}
$$

Therewith and neglecting the transverse momentum due to high doping, we have the basis states

$$
\begin{array}{r}
\Psi_{0,+}^{k, 0}(\mathbf{x}) \approx \frac{e^{i k_{q}(\epsilon) x}}{\sqrt{2}}\left(\begin{array}{c}
1 \\
1
\end{array}\right), \\
\Psi_{0,-}^{k, 0}(\mathbf{x}) \approx \frac{e^{-i k_{q}(\epsilon) x}}{\sqrt{2}}\left(\begin{array}{c}
1 \\
-1
\end{array}\right)
\end{array}
$$

in the leads. Additionally, shifting the Fermi surface of the graphene sheet away from the Dirac point, and thus changing the concentration of carriers, is incorporated into the electrochemical potential $e V_{g}$ of the graphene sheet. For $\left|\epsilon+e V_{g}\right|<\left|\hbar v_{F} q\right|$, we have evanescent modes, ${ }^{52}$ with imaginary $\alpha_{q}(\epsilon)$ and $k_{q}(\epsilon)$. Otherwise, we have propagating modes and scattering is only at $x=0, L$.

Irradiating the two-terminal structure with a laser ${ }^{53}$ can be described by a harmonic ac-bias voltage with driving strength $\alpha=e V_{\mathrm{ac}} / \hbar \omega$ as discovered in the pioneering paper by Tien and Gordon. ${ }^{39}$ Their theory can be incorporated into the scattering formalism, ${ }^{54,55}$ and we are applying it here to the two-terminal graphene structure. In general, incorporating the Tien-Gordon formalism into the scattering approach is not restricted in the driving frequency or amplitude of the ac field if we deal with scattering amplitudes and do not approximate the results in terms of probabilities. But one has to ensure that displacement currents are not dominant and that the quasiparticles have a charge relaxation time within the scattering region that is smaller than the timescale set by the ac-driving frequency. ${ }^{65}$ The importance of displacement currents is determined by the screening properties of the environment of the conductor. The charge relaxation time is given by the $R C$ time $\tau_{R C} \simeq C / G$. It depends on the effective capacitance $C$, which depends on the detailed geometry of the metallic gates around the sample and has to be determined separately for a given experiment. Hence we concentrate below on the particle-current correlation, which can be used to calculate the full electromagnetic response for a given experiment. Taking into account that the exact properties of the nanostructure can vary considerably 
due to imperfections, experimental setups can be in a regime where the Tien-Gordon model is applicable and the results for our graphene setup can serve as a useful starting point for experiment-specific extensions.

We take the two valleys and two pseudospin states of the carbon lattice into account in the prefactor of the current operator of reservoir $\eta$, which reads

$$
\begin{aligned}
\hat{I}_{\eta}(t)= & \frac{2 e W}{\pi \hbar} \sum_{\gamma, \delta=L, R} \sum_{l, k=-\infty}^{\infty} \int_{-\infty}^{\infty} d \epsilon d \epsilon^{\prime} \int_{0}^{\infty} d q J_{l}\left(\alpha_{\gamma}\right) J_{k}\left(\alpha_{\delta}\right) \\
& \times \hat{a}_{\gamma}^{\dagger}(\epsilon-l \hbar \omega) A_{q ; \gamma \delta}\left(\eta, \epsilon, \epsilon^{\prime}\right) \hat{a}_{\delta}\left(\epsilon^{\prime}-k \hbar \omega\right) e^{i\left(\epsilon-\epsilon^{\prime}\right) t / \hbar} .
\end{aligned}
$$

Indices $\gamma, \delta$ run over reservoirs $L, R$. The summation over all modes of $y$ momentum is replaced by an integral since $W \gg L$. Scattering is contained within the current matrix $A_{q ; \gamma \delta}\left(\eta, \epsilon, \epsilon^{\prime}\right)=\delta_{\eta \gamma} \delta_{\eta \delta}-s_{q ; \eta \gamma}^{*}(\epsilon) s_{q ; \eta \delta}\left(\epsilon^{\prime}\right)$ of a current between leads $\gamma$ and $\delta$ measured in lead $\eta$ via the energy-dependent scattering matrix

$$
s_{q}(\epsilon)=\left(\begin{array}{cc}
r_{q}(\epsilon) & t_{q}^{\prime}(\epsilon) \\
t_{q}(\epsilon) & r_{q}^{\prime}(\epsilon)
\end{array}\right) .
$$

The scattering matrix connects in and outgoing scattering states at the two barriers and is calculated in Appendix B by matching the wave functions at $x=0, L$. Here, we write the results for reflection and transmission amplitudes in an alternative version:

$$
\begin{aligned}
& t_{q}(\epsilon)=\frac{2 e^{i k_{q}(\epsilon) L}\left(1+e^{2 i \alpha_{q}(\epsilon)}\right)}{e^{2 i k_{q}(\epsilon) L}\left(1-e^{i \alpha_{q}(\epsilon)}\right)^{2}+\left(1+e^{i \alpha_{q}(\epsilon)}\right)^{2}}, \\
& r_{q}(\epsilon)=\frac{\left(e^{2 i k_{q}(\epsilon) L}-1\right)\left(e^{2 i \alpha_{q}(\epsilon)}-1\right)}{e^{2 i k_{q}(\epsilon) L}\left(1-e^{i \alpha_{q}(\epsilon)}\right)^{2}+\left(1+e^{i \alpha_{q}(\epsilon)}\right)^{2}} .
\end{aligned}
$$

We assume identical scattering for quasiparticles incident from left and right, so $t_{q}(\epsilon)=t_{q}^{\prime}(\epsilon)$ and $r_{q}(\epsilon)=-r_{q}^{\prime}(\epsilon) . r_{q}(\epsilon)$ vanishes if $k_{q}(\epsilon)=\pi n / L$, with integer $n$. The corresponding modes in $y$ direction are determined by

$$
q=\left[\left(\frac{\epsilon}{\hbar v_{F}}\right)^{2}-\left(\frac{\pi n}{L}\right)^{2}\right]^{1 / 2},
$$

giving rise to special features of the current fluctuations, going along with the phase jumps of $\pi L / \hbar v_{F}$ in $r_{q}(\epsilon)$ we discuss later on. At the Dirac point, transmitted quasiparticles at perpendicular incidence perform Klein tunneling via evanescent modes, leading to finite transmission probability $T_{q}(\epsilon)=t_{q}^{\dagger}(\epsilon) t_{q}(\epsilon)$ at small transverse momentum (see Fig. 2).

\section{DIFFERENTIAL CONDUCTANCE}

Since the average current has only a zero-frequency component, PAT events in the conductance ${ }^{17,56}$ can only be studied by inducing photon exchange via a time-dependent voltage as it is, for example, generated by irradiating the setup with a laser beam. Different polarizations of the coupled light field lead to different ac driving in left and right leads. Such an asymmetry can be described by a parameter $a \in[-1,1]$, which varies the driving in the leads via $\alpha_{L / R}=\frac{a \pm 1}{2} \alpha \equiv \frac{V_{\mathrm{ac}, L / R}}{\hbar \omega}$. We call the driving symmetric (in the amplitudes $V_{\mathrm{ac}, L / R}$ ) if $a=0$

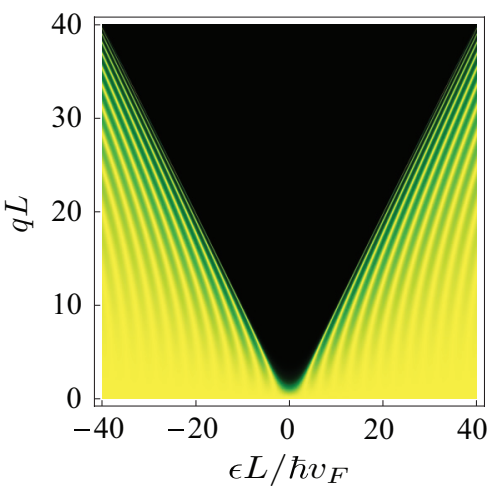

FIG. 2. (Color online) Transmission probability $T_{q}(\epsilon)=\left|t_{q}(\epsilon)\right|^{2}$ as a function of energy and transverse momentum $\mathrm{q}$.

and asymmetric if $a= \pm 1$. For clearness, we will only discuss $a=0, \pm 1$ since intermediate values are just a mixture of those limiting cases. For arbitrary $a$, the differential conductance can be derived from Eq. (15) by taking the statistical and time average and differentiating with respect to voltage. At $k_{B} T=0$, it reads

$$
\begin{aligned}
G(\omega, \alpha)= & \frac{2 e^{2} W}{\hbar} \int_{0}^{\infty} d q \sum_{m=-\infty}^{\infty}\left[J_{m}^{2}\left(\alpha_{L}\right)\left|t_{q}\left(m \hbar \omega+\frac{e V}{2}\right)\right|^{2}\right. \\
& \left.+J_{m}^{2}\left(\alpha_{R}\right)\left|t_{q}\left(m \hbar \omega-\frac{e V}{2}\right)\right|^{2}\right]
\end{aligned}
$$

Different orders $m$ of PAT do not mix but have to be summed up resulting in independent contributions $G_{m}(\omega, \alpha)$ to the differential conductance. Since $G(\omega, \alpha)$ only depends on the Bessel functions squared, these prefactors will always be positive. The influence of the driving strength $\alpha$ on the conductivity $\sigma(\omega, \alpha)=(L / W) G(\omega, \alpha)$ as a function of $\mathrm{dc}$ bias is plotted in Fig. 3. PAT events lead to a substantial enhancement of the conductivity around zero dc bias, because more channels are available in comparison to the case without time-dependent voltages. At large dc-bias voltages, this effect gets negligible since the transmission probability of the graphene sheet, see Fig. 2, is not vanishing at large energies. Thus those contributions built a dominant background. The conductance at arbitrary $\mathrm{dc}$ and ac bias is a sum of two integrated transmission probabilities, where the integrand exhibits crossings of the two independent interference patterns. This behavior is similar to the interference patterns that occur in the discussion of the integrands of the frequency-dependent shot noise, i.e., region III $b$ in the scheme shown in Fig. 4(a), which is explained in the qualitative discussion of Sec. V. Each $G_{m}(\omega, \alpha)$ shows a transition from a region with an oscillating, but in average not increasing contribution to conductance for dc-bias voltages $|e V / 2|<|m \hbar \omega|$, to a regime with a linear increasing background at larger dc-bias voltages. The photon energy $m \hbar \omega$ introduces a phase shift in the oscillations of $G_{m}(\omega, \alpha)$ as a function of dc-bias voltage, so for different $m$ we can have local minima or maxima at $e V=0$. After summation, the conductivity can also show a local minimum or maximum at $e V=0$, as it can be observed for the various values of $\alpha$ in Fig. 3(a). If $|a|$ tends to one, this effect is hidden behind the contribution from the terminal where driving gets 

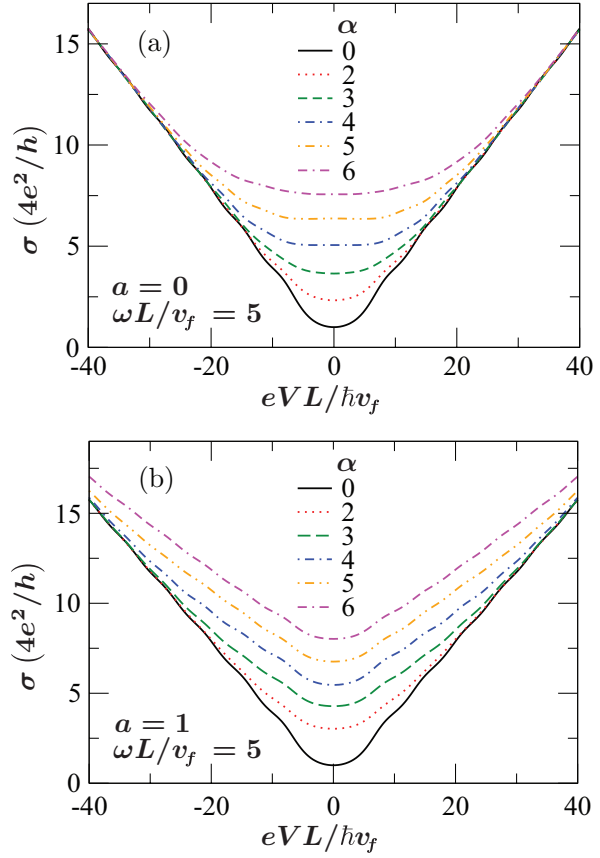

FIG. 3. (Color online) Conductivity $\sigma(\omega, \alpha)=(L / W) G(\omega, \alpha)$ as a function of de voltage applied across the two-terminal setup. We show curves for various ac-driving strengths $\alpha$ applied to (a) both reservoirs $(a=0)$ and (b) to the left reservoir only $(a=1)$ with $\omega L / v_{f}=5$.

small, as in Fig. 3(b) with $a=1$. From the oscillations with a period proportional to $L$, we expect no measurable effect on the conductivity or the shot noise, ${ }^{22,24}$ as in the case without ac driving and for the zero-frequency Fano factor. In the scattering approach, they are simply because the transmission function oscillates as a function of energy. But imperfections of real samples, as impurities ${ }^{57}$ or lattice mismatch, lead to scattering events. Due to this randomizing effect on the path lengths for propagating quasiparticles, the calculated oscillations are averaged out in experiment. ${ }^{22,24}$

\section{FREQUENCY-DEPENDENT SHOT NOISE}

To get full information on current-current correlations, we study the nonsymmetrized noise spectrum as it can be detected by an appropriate measurement device in the quantum regime. ${ }^{58-74}$ We allow harmonic ac driving $e V_{\text {ac }} \cos (\omega t)$ in the
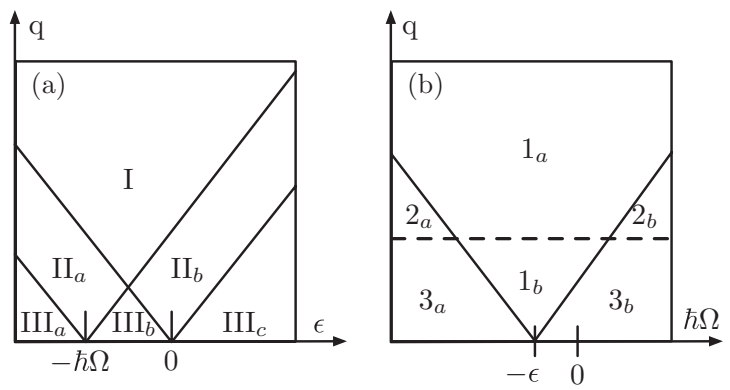

FIG. 4. Schematic view of the different regions, which occur in the integrands of the correlators contributing to the finite-frequency shot noise spectrum. leads, so in Fourier space, the current-current correlations are defined as

$$
S_{\alpha \beta}\left(\Omega, \Omega^{\prime}, \omega\right)=\int_{-\infty}^{\infty} d t d t^{\prime} S_{\alpha \beta}\left(t, t^{\prime}, \omega\right) e^{i \Omega t+i \Omega^{\prime} t^{\prime}} .
$$

The nonsymmetrized shot noise correlates currents at two times:

$$
S_{\alpha \beta}\left(t, t^{\prime}, \omega\right)=\left\langle\Delta \hat{I}_{\alpha}(t) \Delta \hat{I}_{\beta}\left(t^{\prime}\right)\right\rangle
$$

with variance $\Delta \hat{I}_{\alpha}(t)=\hat{I}_{\alpha}(t)-\left\langle\hat{I}_{\alpha}(t)\right\rangle$. Of experimental interest are the fluctuations on time scales large compared to the one defined by the driving frequency $\omega$. Thus, as in Ref. 54, we introduce Wigner coordinates $t=T+\tau / 2$ and $t^{\prime}=T-\tau / 2$ and average over a driving period $2 \pi / \omega$. Then, the noise spectrum is defined by the quantum statistical expectation value of the Fourier-transformed current operator $\hat{I}_{\alpha}(\Omega)$ via $S_{\alpha \beta}\left(\Omega, \Omega^{\prime}, \omega\right)=2 \pi S_{\alpha \beta}(\Omega, \omega) \delta\left(\Omega+\Omega^{\prime}\right)=\left\langle\hat{I}_{\alpha}(\Omega) \hat{I}_{\beta}\left(\Omega^{\prime}\right)\right\rangle$. $S_{\alpha \beta}(\Omega, \omega)$ is nothing but the Fourier transform of $S_{\alpha \beta}(\tau, \omega)$. Similarly, in the case without ac driving, the noise is only a function of relative times $\tau=t-t^{\prime}$. In order to keep the notation short, in the de limit, we write $S_{\alpha \beta}(\Omega):=S_{\alpha \beta}(\Omega, \omega=$ $0)$. To get a deeper insight into the underlying processes of charge transfer, we split the noise into four possible correlators $^{61}$ defined by

$$
S_{L L}(\Omega, \omega):=\sum_{\alpha, \beta=L, R} C_{\alpha \rightarrow \beta}(\Omega, \omega) .
$$

The correlators themselves can be seen as the building blocks of noise spectra where different combinations describe noise detected by corresponding measurement setups. ${ }^{60,61}$ First, we discuss $S_{L L}(\Omega):=S_{L L}(\Omega, \omega=0)$, the case when no ac driving is present. We also skip $\omega$ in the arguments of the correlators. Then an evaluation of Eq. (21) at $k_{B} T=0$ leads to the expressions

$$
\begin{aligned}
& C_{L \rightarrow L}(\Omega) \\
& =\frac{e^{2} \Theta(\hbar \Omega)}{2 \pi \hbar} \int_{\mu_{L}-\hbar \Omega}^{\mu_{L}} d \epsilon \int_{-\infty}^{\infty} d q\left|r_{q}^{*}(\epsilon) r_{q}(\epsilon+\hbar \Omega)-1\right|^{2}, \\
& C_{R \rightarrow R}(\Omega) \\
& \quad=\frac{e^{2} \Theta(\hbar \Omega)}{2 \pi \hbar} \int_{\mu_{R}-\hbar \Omega}^{\mu_{R}} d \epsilon \int_{-\infty}^{\infty} d q T_{q}(\epsilon) T_{q}(\epsilon+\hbar \Omega), \quad(24 \mathrm{~b}) \\
& C_{L} \rightarrow R(\Omega) \\
& \quad=\frac{e^{2} \Theta(\hbar \Omega-e V)}{2 \pi \hbar} \int_{\mu_{R}-\hbar \Omega}^{\mu_{L}} d \epsilon \int_{-\infty}^{\infty} d q R_{q}(\epsilon) T_{q}(\epsilon+\hbar \Omega), \\
& C_{R \rightarrow L}(\Omega) \\
& \quad=\frac{e^{2} \Theta(\hbar \Omega+e V)}{2 \pi \hbar} \int_{\mu_{L}-\hbar \Omega}^{\mu_{R}} d \epsilon \int_{-\infty}^{\infty} d q T_{q}(\epsilon) R_{q}(\epsilon+\hbar \Omega) .
\end{aligned}
$$

At finite dc-bias voltages, correlations with initial and final states related to the measurement terminal $L$ are special in the sense that they can not be written in terms of probabilities at finite frequency. For symmetrized noise, Büttiker ${ }^{76}$ discussed the essential role of the complex reflection amplitudes in elastic 
electron transport and how they determine the equilibrium current fluctuations. In the quantum regime at $k_{B} T=0$, the equilibrium fluctuations are given by

$$
\begin{aligned}
S_{L L}(\Omega)= & \frac{e^{2}}{2 \pi \hbar} \Theta(\hbar \Omega) \int_{-\hbar \Omega}^{0} d \epsilon \int_{-\infty}^{\infty} d q\left[2-r_{q}^{*}(\epsilon) r_{q}(\epsilon+\hbar \Omega)\right. \\
& \left.-r_{q}^{*}(\epsilon+\hbar \Omega) r_{q}(\epsilon)\right] .
\end{aligned}
$$

For finite dc bias, the reflection amplitudes entering $C_{L \rightarrow L}(\Omega)$ play the same essential role as in equilibrium, in the sense that finite-frequency current fluctuations are nonzero even for vanishing transmission. The combination of scattering matrices of the correlators integrands, which enter in the current-current cross-correlation spectrum,

$$
S_{L R}(\Omega, \omega):=\sum_{\alpha, \beta=L, R} C_{\alpha \rightarrow \beta}^{\mathrm{c}}(\Omega, \omega),
$$

are substantially different than in the ones for the autoterminal noise. Most of all, at finite frequency, none of the complex correlators can be written as an integral over transmission or reflection probabilities:

$$
\begin{aligned}
C_{L \rightarrow L}^{\mathrm{c}}(\Omega)= & \frac{e^{2} \Theta(\hbar \Omega)}{2 \pi \hbar} \int_{\mu_{L}-\hbar \Omega}^{\mu_{L}} d \epsilon \int_{-\infty}^{\infty} d q t_{q}^{*}(\epsilon+\hbar \Omega) t_{q}(\epsilon) \\
& \times\left[1-r_{q}^{*}(\epsilon) r_{q}(\epsilon+\hbar \Omega)\right], \\
C_{R \rightarrow R}^{\mathrm{c}}(\Omega)= & \frac{e^{2} \Theta(\hbar \Omega)}{2 \pi \hbar} \int_{\mu_{R}-\hbar \Omega}^{\mu_{R}} d \epsilon \int_{-\infty}^{\infty} d q t_{q}^{*}(\epsilon) t_{q}(\epsilon+\hbar \Omega) \\
& \times\left[1-r_{q}^{*}(\epsilon+\hbar \Omega) r_{q}(\epsilon)\right], \\
C_{L \rightarrow R}^{\mathrm{c}}(\Omega)= & \frac{-e^{2} \Theta(\hbar \Omega-e V)}{2 \pi \hbar} \int_{\mu_{R}-\hbar \Omega}^{\mu_{L}} d \epsilon \int_{-\infty}^{\infty} d q r_{q}^{*}(\epsilon) \\
& \times t_{q}(\epsilon) r_{q}^{*}(\epsilon+\hbar \Omega) t_{q}(\epsilon+\hbar \Omega), \\
C_{R \rightarrow L}^{\mathrm{c}}(\Omega)= & \frac{-e^{2} \Theta(\hbar \Omega+e V)}{2 \pi \hbar} \int_{\mu_{L}-\hbar \Omega}^{\mu_{R}} d \epsilon \int_{-\infty}^{\infty} d q t_{q}^{*}(\epsilon) \\
& \times r_{q}(\epsilon) t_{q}^{*}(\epsilon+\hbar \Omega) r_{q}(\epsilon+\hbar \Omega) .
\end{aligned}
$$

Unlike for symmetrized noise, quantum noise ${ }^{65,72}$ spectra discriminate between photon absorption $(\Omega>0)$ and emission $(\Omega<0)$ processes between quasiparticles in graphene and a coupled electric field. ${ }^{60,61,73-75}$ The energy for photon emission has to be provided by the voltage source, so at $k_{B} T=0$ the Heaviside $\Theta$ functions ensure that only terms satisfying this condition contribute at negative frequencies. In the dc limit, our choice of chemical potentials $-\mu_{L}=\mu_{R}=e V / 2>0$ and the fact that the measurement is performed at reservoir $L$, leaves only $C_{R \rightarrow L}^{\mathrm{c}}(\Omega) \neq 0$ if $\Omega \leqslant 0$. When additional ac voltages are present none of the correlators of Eq. (A1) is given in terms of probabilities and integration boundaries are changed by $\pm m \hbar \omega$. Then all correlators can contribute at frequencies $\Omega<0$.

\section{QUALITATIVE DISCUSSION}

A good starting point to interpret the results for the conductivity and shot-noise spectra is to examine the involved integrands in Eqs. (24) and (27). Figure 4 provides a schematic overview of the different regions occurring in the $2 \mathrm{D}$ plots of Figs. 5-10. We show the real parts of integrands either as a function of $(q, \epsilon)$ as in scheme $4(\mathrm{a})$ or of $(q, \Omega)$ as in
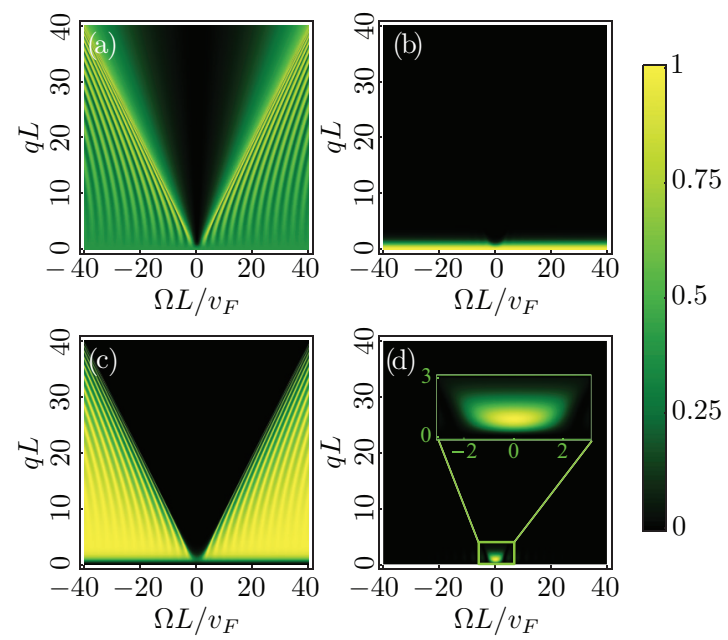

FIG. 5. (Color online) Real parts of integrands of the four correlators [see Eq. (24)] contributing to the shot noise, namely, (a) $0.25\left|1-r_{q}^{*}(\epsilon) r_{q}(\epsilon+\hbar \Omega)\right|^{2}$, (b) $T_{q}(\epsilon) T_{q}(\epsilon+\hbar \Omega)$, (c) $R_{q}(\epsilon) T_{q}(\epsilon+$ $\hbar \Omega$ ), and (d) $R_{q}(\epsilon+\hbar \Omega) T_{q}(\epsilon)$. Here, the energy is fixed at $\epsilon=0$ corresponding to vanishing dc bias. The correlator in (a) cannot be written in terms of probabilities, except in the zero-frequency limit the integrand results in $T_{q}^{2}(\epsilon)$. Correlator (b) contains one transmission probability at zero energy that is only nonzero at small $q$. Since for small transversal momentum $R_{q}(\epsilon)$ decays as $q^{-2}$, the correlator (c) tends to zero in this regime and otherwise mimics the behavior of $T_{q}(\epsilon)$. Integrand (d) is also restricted to low transverse momentum because $T_{q}(\epsilon)=0$ otherwise.

scheme 4(b). The former is divided by the four envelopes $q=|\epsilon|$ and $\hbar v_{F} q=|\epsilon+\hbar \Omega|$ into six areas: (1) I, (2) where the regimes $\mathrm{II}_{a}$ and $\mathrm{II}_{b}$ of evanescent modes are merging, and (3) the areas $\mathrm{III}_{a}, \mathrm{III}_{a}, \mathrm{III}_{a}$ of propagating modes. Area $\mathrm{III}_{b}$ is defined by the two lines with origins $(q=0, \epsilon=0),(q=$ $0, \epsilon=-\hbar \Omega)$ and intersection $\left(\hbar v_{F} q=\hbar \Omega / 2, \epsilon=-\hbar \Omega / 2\right)$. Areas in scheme 4(b) are separated by $\hbar v_{F} q=|\epsilon+\hbar \Omega|$ and the dashed horizontal line $\hbar v_{F} q=|\epsilon|$. The transmission probability fits into this scheme when the horizontal separation is absent so we are left with areas $1_{a}$ and $2_{a / b}$. Then area $1_{a}$ includes the black region of Fig. 2 where no transmission is possible, and the regime of evanescent modes with finite transmission probability for small $|\epsilon|<\hbar v_{F}|q|$ around $\epsilon=0$ due to Klein tunneling. In regimes $2_{a / b}$, a hyperbolic shaped interference pattern with oscillations along $\epsilon$ is prominent, where the period of oscillations is on the order of $\hbar v_{f} / L$ for small $\hbar v_{F}|q| \ll|\epsilon|$. Figure 5 shows the relevant integrands of the four correlators $C_{\alpha \rightarrow \beta}(\Omega)$ contributing to the finite frequency quantum noise, plotted as a function of $(q, \Omega)$ when $\epsilon=0$. Then the imaginary part of $r_{q}^{*}(\epsilon) r_{q}(\epsilon+\hbar \Omega)$ leads to finite contributions in the region $\mathrm{I}_{a}$ and $\mathrm{I}_{b}$ in Fig. 5(a). $T_{q}(\epsilon=0)$ is only nonzero for small $q$, so integrands (b) and (d) vanish for large $q$. Since $R_{q}(\epsilon)=1-T_{q}(\epsilon)$, integrand (c) vanishes when $q \rightarrow 0$ and otherwise resembles the shape of $T_{q}(\epsilon)$.

A finite $\epsilon$, as in Fig. 6, introduces another interference pattern for propagating modes. In region $1_{a}$, nonzero values are possible, and in $2_{a}$ and $2_{b}$, the usual interferences occur. For $q$ values below $\hbar v_{F}|q|=|\epsilon|$, this additional pattern can be seen in region $1_{b}$. The interplay of both patterns leads to phase 

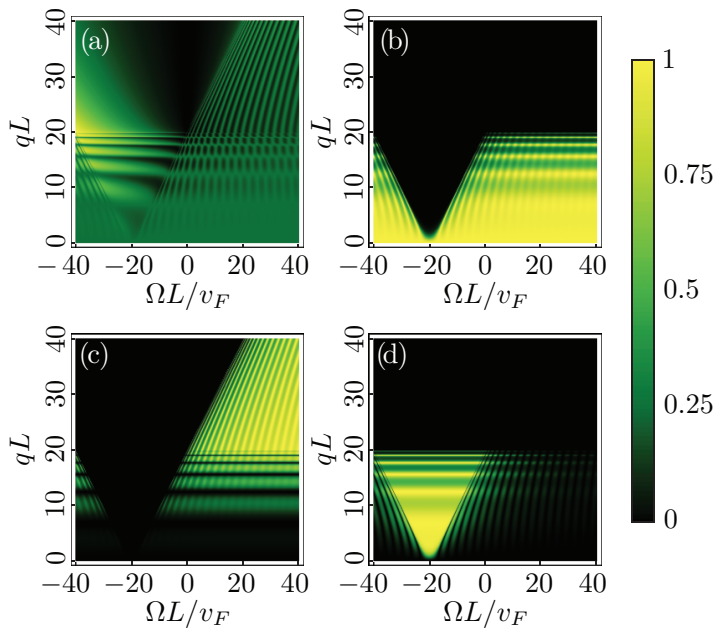

FIG. 6. (Color online) Real parts of integrands of correlators [see Eq. (24), see also Fig. 5] contributing to the shot noise for fixed energy $\epsilon L / \hbar v_{F}=20$. At finite $\epsilon$, there is an additional interference pattern along $q$ if $\hbar v_{F}|q|<|\epsilon|$, leading to phase jumps in the integrand of correlator (a), the one where initial and final states belong to the measurement terminal $L$. When the integrand can be written as a product of probabilities, see (b)-(d), the phase jumps are absent but two independent interference patterns are found.

jumps of $\pi L / \hbar v_{F}$ in regions $3_{a}$ and $3_{b}$. These phase jumps can be determined by requiring $\left|r_{q}^{*}(\epsilon) r_{q}(\epsilon+\hbar \Omega)-1\right|^{2}=1$ in Eq. (24a), see Fig. 6(a). This requirement is fulfilled when $r_{q}^{*}(\epsilon) r_{q}(\epsilon+\hbar \Omega)$ vanishes, what is fulfilled by the transversal momenta of Eq. (19). The condition $\left|r_{q}^{*}(\epsilon) r_{q}(\epsilon+\hbar \Omega)-1\right|^{2}=$ 4 for a maximum in the integrand leads to modes that experience Klein tunneling. Actually, this correlator can be written as integral over $1+R_{q}(\epsilon) R_{q}(\epsilon+\hbar \Omega)-2\left[R_{q}(\epsilon) R_{q}(\epsilon+\right.$ $\hbar \Omega)]^{1 / 2} \cos [\Phi(\epsilon, \Omega)]$ including a scattering phase $\Phi(\epsilon, \Omega)=$
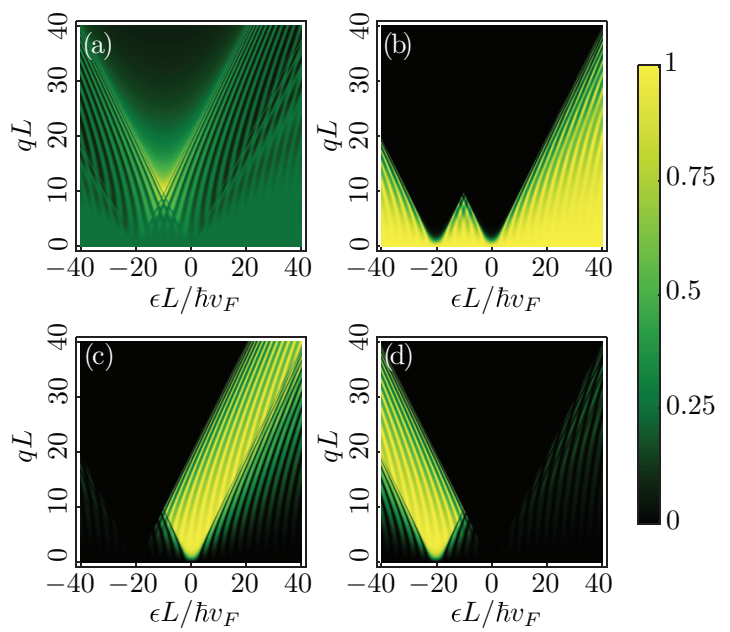

FIG. 7. (Color online) Real parts of integrands of the correlators [see Eq. (24), see also Fig. 5] contributing to the shot noise with fixed frequency $\Omega L / v_{F}=20$. Analogous to Fig. 6 but as a function of $(q, \epsilon)$. Phase jumps occur in the interval $-\hbar \Omega<\epsilon<0$ in integrand (a), region $\mathrm{III}_{b}$ of Fig. 4(a). The interplay of the two interference patterns can also be observed at larger energies and transverse momenta for $\hbar v_{F}|q|<|\epsilon|, \hbar v_{F}|q|<|\epsilon+\hbar \Omega|$ in all integrands (a)-(d).
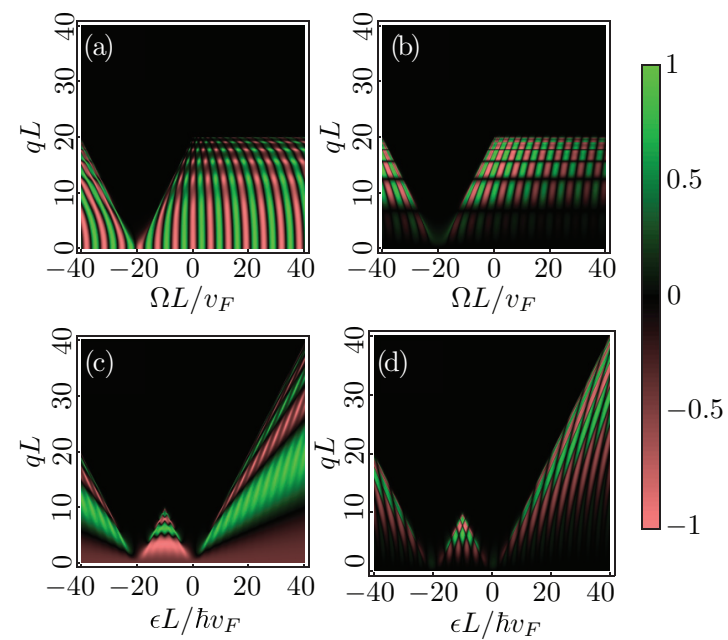

FIG. 8. (Color online) Real parts of integrands that appear in Eq. (27) contributing to the cross-correlation shot noise for fixed energy $\epsilon L / \hbar v_{F}=20$ (top) and fixed frequency $\Omega L / v_{F}=20$ (bottom), namely, (a) and (c) $\Re\left\{t_{q}^{*}(\epsilon+\hbar \Omega) t_{q}(\epsilon)\left[r_{q}^{*}(\epsilon+\hbar \Omega) r_{q}(\epsilon)-1\right]\right\}$ and (b) and (d) $4 \Re\left[r_{q}^{*}(\epsilon) t_{q}(\epsilon+\hbar \Omega) r_{q}^{*}(\epsilon+\hbar \Omega) r_{q}(\epsilon)\right]$. Due to symmetry reasons, the integrands are identical when interchanging index labels $L, R$. As a function of frequency integrand (a) leads to strongly oscillating contributions to the noise spectrum. These oscillations are reduced due to the alternating behavior along $q$ in cross-terminal contributions (b). In (c) and (d), the integrands are plotted as a function of $(q, \epsilon)$ where they reveal a similar structural difference.

$\operatorname{Arg}\left[r_{q}^{*}(\epsilon) r_{q}(\epsilon+\Omega)\right]$. Thus it describes events containing the scattering phase between time-reversed paths of electron-hole pairs separated by the photon energy $\hbar \Omega$ reflected back into the measurement terminal. The effect of the phase shifts on
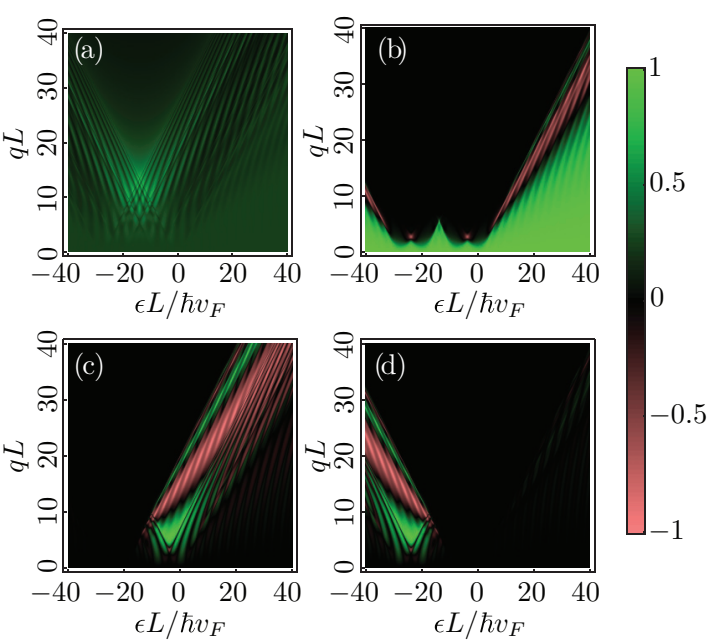

FIG. 9. (Color online) Real parts of integrands appearing in the correlators of Eq. (A1) contributing to the shot noise for driving frequency $\omega L / v_{F}=7.5$ and fixed frequency $\Omega L / v_{F}=20$. The integrands are (a) $0.25\left[1-r_{q}^{*}(\epsilon) r_{q}(\epsilon+\hbar \Omega)\right]\left[1-r_{q}^{*}(\epsilon+\hbar \Omega+\right.$ $\left.\hbar \omega) r_{q}(\epsilon+\hbar \omega)\right]$, (b) $t_{q}^{*}(\epsilon) t_{q}(\epsilon+\hbar \Omega) t_{q}^{*}(\epsilon+\hbar \Omega+\hbar \omega) t_{q}(\epsilon+\hbar \omega),(\mathrm{c})$ $r_{q}^{*}(\epsilon) t_{q}(\epsilon+\hbar \Omega) t_{q}^{*}(\epsilon+\hbar \Omega+\hbar \omega) r_{q}(\epsilon+\hbar \omega)$, and (d) $t_{q}^{*}(\epsilon) r_{q}(\epsilon+$ $\hbar \Omega) r_{q}^{*}(\epsilon+\hbar \Omega+\hbar \omega) t_{q}(\epsilon+\hbar \omega)$. When two frequencies are present none of the correlators can be written in terms of probabilities and additional phase jumps come into play. 

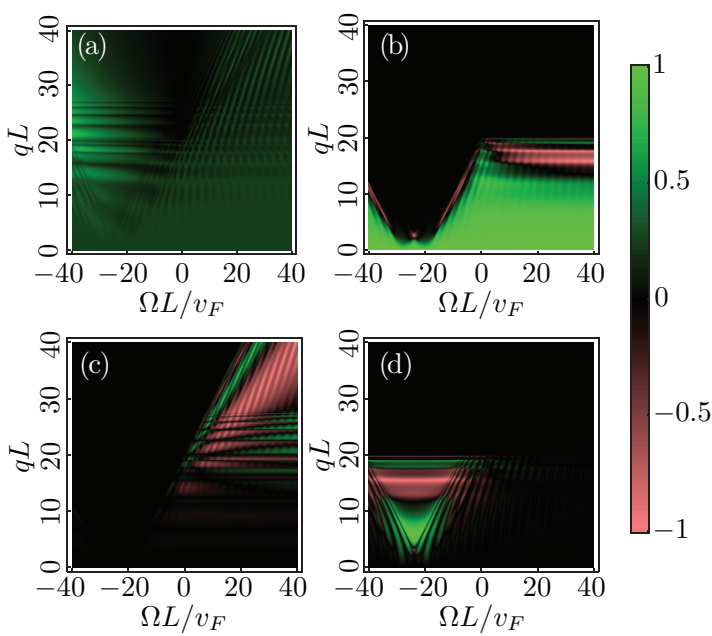

FIG. 10. (Color online) Real parts of integrands of the correlators contributing to the shot-noise with driving frequency $\omega L / v_{F}=7.5$ and fixed energy $\epsilon L / \hbar v_{F}=20$. Analogous to Fig. 9, but as a function of $(q, \Omega)$. As a consequence of PAT, horizontal interference lines occur for transverse momenta $\hbar v_{F}|q|<|\epsilon+m \hbar \omega|$ as in (a) and (c).

the integrands interference patterns is also obvious in the $(q, \epsilon)$ plot of Fig. 7(a), region $\mathrm{III}_{b}$. Figures 7(b)-7(d) show a similar interference pattern although the corresponding correlators are defined in terms of probabilities.

Concerning cross-correlation noise, the integrands occurring in Eq. (27) show alternating patterns of positive and negative values. The ones that describe autoterminal contributions to $S_{L R}(\Omega)$ [Eqs. (27a) and (27b)], as in Fig. 8(a), have an alternating sign along $\Omega$. In the cross-terminal ones [Eqs. (27c) and (27d)], as in Fig. 8(b), the additional interference pattern along $q$ introduces another change of sign. Figures 8(c) and 8(d) show a similar behavior for the dependence on $(q, \epsilon)$. When ac-bias voltages introduce the driving frequency $\omega$, the integrands structures become even richer but also less clear, as in Figs. 9 and 10. Then alternating signs in all contributions to autocorrelation noise are observed, except for the correlator with initial and final sates in the measurement terminal. This results in peculiar oscillatory features in the interference patterns at combinations of all involved energies $\epsilon, \hbar \Omega, m \hbar \omega$. Predicting the effect of such features on the noise spectra from the plotted integrands is then almost impossible because one still has to average over all possible energies and $q$ values by integration.

\section{AUTOCORRELATION NOISE}

In contrast to the conductivity, the shot-noise spectrum, in general, couples different orders of PAT events, expressed by the product of four Bessel functions of arbitrary order. But since the driving is fixed, nonvanishing contributions exist only up to a certain order depending on the precise value of $\alpha$. When time-dependent voltages are present, current fluctuations of Eq. (A1) contain products of four scattering matrices, each with a different energy argument. After performing the dc-bias limit, only transitions between $\epsilon$ and $\epsilon+\hbar \Omega$ are left.

\section{A. Shot-noise spectrum}

In the regime $e V, \hbar \Omega, \hbar \omega \ll \hbar v_{F} / L$, the scattering matrix can be treated as energy independent. Then, as for a single level quantum dot in the broadband limit, the asymmetric quantum noise as a function of frequency is the sum of four straight lines, with kinks at $\hbar \Omega=0, \pm e V \cdot{ }^{60,61}$ For vanishing dc bias, we have $C_{R \rightarrow L}(\Omega)=C_{L \rightarrow R}(\Omega)$ and $C_{R \rightarrow L}(\Omega) \approx C_{L \rightarrow R}(\Omega)$, as long as $\Omega \ll v_{F} / L$. The richer regime, when $e V, \hbar \Omega, \hbar \omega>$ $\hbar v_{F} / L$, additionally exhibits strongly oscillating integrands. Those oscillations are purely due to propagating modes as it is also clear from interference patterns of the integrands in Figs. 5-9, regions $\mathrm{II}_{a, b}$ and $\mathrm{III}_{a, b, c}$. In the special case of perpendicular incidence $\left[q, \alpha_{q}(\epsilon)=0\right]$, we have Klein tunneling, thus the frequency dependence of the correlators is linear for this mode. Then $C_{\alpha \rightarrow \beta}(\Omega)=0$ if $\alpha \neq \beta$ since $R_{q}(\epsilon)=0$, otherwise, the $C_{\alpha \rightarrow \beta}(\Omega)$ mirror the interference patterns of the integrands. So the noise spectrum (see Fig. 11
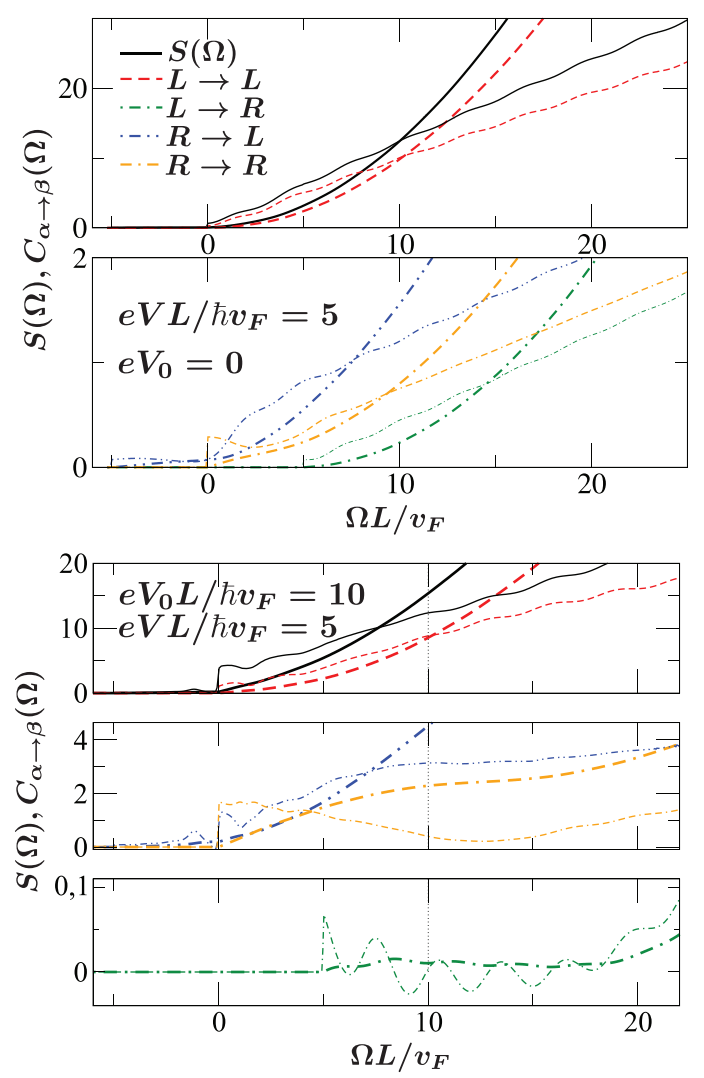

FIG. 11. (Color online) Real parts of autocorrelation noise spectrum in units of $2 \pi \hbar / e^{2}$. We compare a setup where dc-bias voltages are fixed symmetrically around the Dirac point (top,) with the case when $e V_{0} L / \hbar v_{F}=2 e V / \hbar v_{F}=10$ (bottom). Thick lines are shot noise and correlators. Thin lines are derivatives with respect to frequency. Contributions from $C_{L \rightarrow L}(\Omega)$ are dominant at positive frequencies. (Top) Special features in the derivatives are seen for frequencies $\hbar \Omega<e V$ in the $R \rightarrow R$ contribution, when the lower bound of the energy-integration interval approaches the Dirac point (compare to Figs. 6 and 7). (Bottom) The distance to the Dirac point is increased by the offset voltage. Therefore oscillatory features appear in a larger frequency interval and in all four correlators, since integration boundaries in all contributions are crossing the Dirac point with increasing $\Omega$. 
solid, thick curve) shows oscillations on the scale of $L / \hbar v_{F}$ in the regime $e V, \hbar \Omega, \hbar \omega \gg \hbar v_{F} / L$, similar to the shot noise at zero frequency as a function of the electrochemical potential in the graphene sheet. ${ }^{20}$ Although present in all four correlators, the oscillations show up in the noise spectrum mainly via $C_{L \rightarrow L}(\Omega)$ of the terminal where the fluctuating currents are probed. That is because the correlator itself, as well as the amplitude of the oscillations, are significantly larger than for other contributions. Therefore, in comparison to the absorption branch (positive frequencies), the emission branch of the spectrum (negative frequencies) shows only small shot noise. Indeed, all correlators except $C_{R \rightarrow L}(\Omega)$ vanish when $\Omega \leqslant 0$ since the energy for the emission of a photon has to be provided by the voltage source. Especially, the contribution dominant at positive frequencies vanishes: $C_{L \rightarrow L}(\Omega)=0$ if $\hbar \Omega \leqslant 0$.

We are considering the limit $k_{B} T=0$ where the correlators integration windows are exactly determined by the chemical potentials. At finite temperature, this so-defined onsets of the four contributions as a function of frequency are smeared out by the broadening of the Fermi functions. Clearly, a shift of the electrochemical potential in the graphene sheet does not affect these onsets since it does not enter in the Fermi functions of the leads, but it still changes the transmission function resulting in a modified spectrum. Those limits of energy integration, as well as their position relative to region $\mathrm{III}_{b}$, result in features in the noise spectra besides the discussed oscillations. In order to clarify the role of the Dirac Hamiltonian in comparison to the role of pure Fabry-Pérot interferences, we compare results when the charge injection is only in the conduction or valence band by shifting the dc-bias voltages above the Fermi energy of the graphene sheet via the offset voltage $V_{0}$ in $\mu_{L / R}= \pm e V / 2+e V_{0} . C_{L \rightarrow R}(\Omega)$ can never see the regime $-\hbar \Omega<\epsilon<0$ when $e V_{0}=0$, as in the top of Fig. 11. Thus the oscillations visible in the derivative have a well defined period over the whole spectrum on top of a linearly increasing background. When an offset voltage $e V_{0}=2 e V$ is applied, as done when calculating the spectra for the lower plot of Fig. 11, $C_{R \rightarrow R}(\Omega)$ shows a complicated frequency dependence for small $\Omega$. The contribution $C_{L \rightarrow L}(\Omega)$ describes correlations of scattering states emanating from the left reservoir reflected back into the same reservoir. We will discuss this contribution now in detail. The special features for small frequency are due to the interplay of the integration boundaries with the various regions in Fig. 4(a) occurring in the integrands $(q, \epsilon)$ dependence of Fig. 7(a). The integration is over all $q$ modes and from $\epsilon=-e V / 2+e V_{0}-\hbar \Omega$ to $\epsilon=e V_{0}-e V / 2$. When $e V_{0}=0, e V=0$, this corresponds to $-\hbar \Omega<\epsilon<0$, regions $\mathrm{III}_{b}$ and partly $\mathrm{II}_{a, b}$ of Fig. 4(a). Now at finite $e V, e V_{0}$ as in Fig. 11, the integration window can include region $\mathrm{III}_{b}$ completely, partly, or not at all, resulting in variations of the spectrum. At small $\hbar \Omega$, features in the integrands interference patterns have stronger impact. This can be seen from strongly nonharmonic features of the noise spectrum, e.g., in $C_{L \rightarrow L}(\Omega)$ and $C_{R \rightarrow L}(\Omega)$ for $e V_{0}=2 e V$. For large frequencies, averaging leads to nearly harmonic oscillations on top of the increasing background. With the chosen parameters, the distance of the chemical potential $\mu_{L}$ to the charge-neutrality point is given by $e\left(-V / 2+V_{0}\right) L /\left(\hbar v_{F}\right)=7.5$. Around the corresponding frequency, the oscillatory behavior of the spectrum is modified and flattened due to a reduced fraction of propagating modes.
Raising the frequency further increases this fraction again and oscillations are roughly harmonic with period $\pi L / \hbar v_{F}$, best visible in the derivatives $d C_{L \rightarrow L}(\Omega) / d \Omega$ of Fig. 11 . That is also the point where the lower bound of energy integration starts to include the special interference pattern of the integrands around the energy interval $-\Omega<\epsilon<0$, region $\mathrm{III}_{b} . C_{R \rightarrow R}(\Omega)$ is not influenced by the measurement terminal itself, but probes transmission probabilities via scattering events that are related to the right terminal only. An analogous behavior of the spectrum as before is found, this time with a distance $e\left(V / 2+V_{0}\right) L /\left(\hbar v_{F}\right)=12.5$ of the lower integration boundary to the charge neutrality point when $\hbar \Omega=0$. Now increasing frequency is going along with a decreasing slope of the derivative with respect to frequency until the Dirac point is reached. There, the slope increases again since more open channels become available. The same interpretation also explains features in the interval $\hbar \Omega<e V$ of the autoterminal correlators shown in Fig. 11, when $V_{0}=0$. For example, the spectrum of the correlator (24b), with initial and final states in the right lead, exhibits a reducing slope until $\hbar \Omega=e V / 2$ from where on the oscillations have a well-defined period. The $d C_{R \rightarrow R}(\Omega) / d \Omega$ curve has a maximal slope at $\hbar \Omega=e V$ when positive and negative energies with same magnitude are present. For higher frequencies, oscillations have again a well-defined phase.

We also study the excess noise at finite frequencies: $S_{\mathrm{exc}}(\Omega, \omega):=\left.S(\Omega, \omega)\right|_{e V}-\left.S(\Omega, \omega)\right|_{e V=0}$. Subtracting the noise at zero bias voltage removes the divergent contributions from the noise spectrum. Then oscillating features due to bias-voltages are more obvious since they are now also prominent in the noise spectra of Fig. 12, not only in derivatives. When $e V_{0}=0$ the excess noise (thick, black, solid curve) is purely positive for $\hbar \Omega \ll e V$, while for $\hbar \Omega>e V$, it is oscillating around zero, because then cross-terminal contributions $C_{\alpha \rightarrow \beta}(\Omega)$ cancel each other up to a constant offset acquired at small $\Omega$. This offset is compensated by the $L \rightarrow L$ contribution. Oscillations of this contribution have again a considerable impact on the excess noise spectrum. In the lower plot of Fig. 12, the offset voltage is fixed to $e V_{0}=2 e V$. For low frequencies $\hbar \Omega<e V$, complicated oscillations occur in all contributions to excess noise and are accompanied by a strongly increasing slope up to frequencies $\hbar \Omega>e V_{0}+e V / 2$. As for the noise itself, the frequency of the oscillations is determined by $\hbar \Omega_{Z}=2 e V$ and equals the frequency expected from the Zitterbewegung of relativistic Dirac fermions. ${ }^{12}$ This frequency corresponds to a period of $\pi$ in our plots. It would be interesting to test experimentally if those much more pronounced oscillations, compared to the overall shot noise, can be detected in spite of randomization effects of imperfections on the quasiparticles path lengths. In summary, (i) the impact of the Dirac Hamiltonian on the frequency dependence of autoterminal current fluctuations leads to peculiar oscillation for energies in the vicinity of the Dirac point as an interplay of Klein tunneling, phase jumps in the correlators, and their energy-integration limits. (ii) Oscillations due to the FP setup have a constant phase for high energies when propagating modes are dominant. Then $d S^{\operatorname{exc}}(\Omega) / d \Omega$ oscillates between positive and negative values with a period as expected from the effect of Zitterbewegung. 

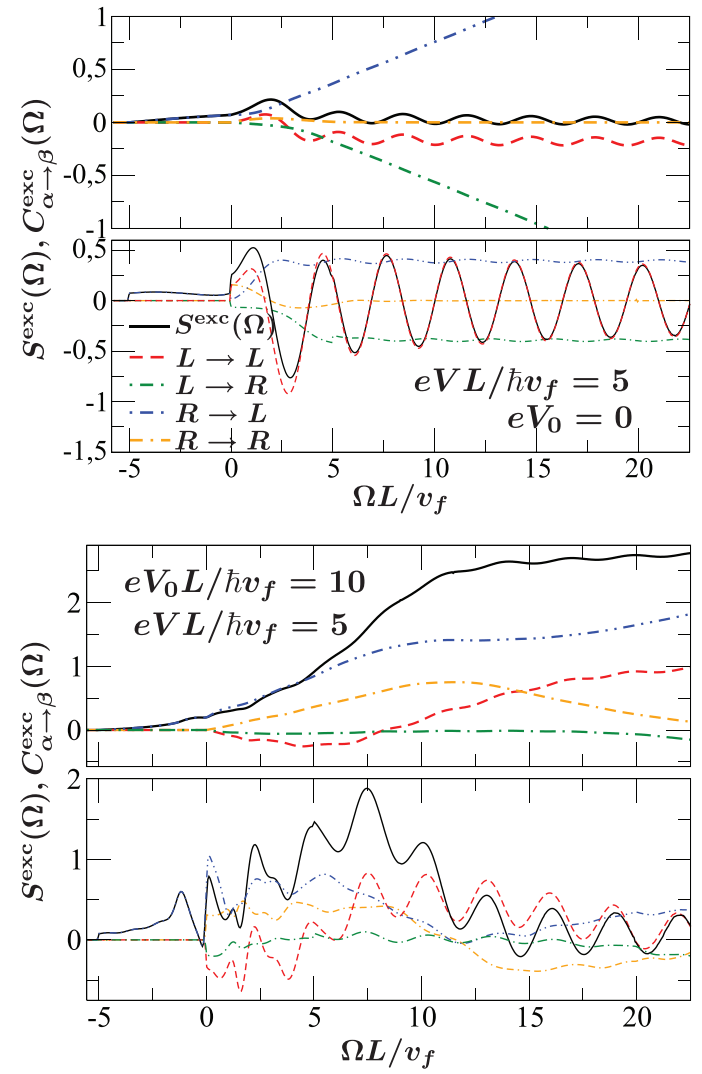

FIG. 12. (Color online) Real parts of autocorrelation excess-noise spectrum in units of $2 \pi \hbar / e^{2}$ (thick lines, top) and derivatives (thin lines, bottom) with dc bias symmetrically applied around the Dirac point (top) and for finite $e V_{0}=2 e V$ (bottom). By subtracting the noise at zero dc bias, the divergent background is removed. The structure and especially the oscillatory behavior are coined by autoterminal contributions of Eq.(27a) related to the measurement terminal $L$. The jump in the derivative of $C_{L \rightarrow R}(\Omega)$ is present because this correlator does not contribute for frequencies $\hbar \Omega<e V$. By applying an offset voltage $e V_{0} L / \hbar v_{F}=10$ a complicated structure emerges, best visible in the derivatives.

\section{B. Dc-bias dependence at finite frequency}

Analogous to the spectrum, the dc-bias dependence for fixed frequency is featureless in the regime $e V, \hbar \Omega, \hbar \omega \ll$ $\hbar v_{F} / L$, except the pronounced onsets of the four correlators. This is not surprising when looking at the derivatives with respect to voltage:

$$
\begin{aligned}
\frac{d C_{L \rightarrow L}}{d V}= & \frac{e^{2} \Theta(\Omega)}{4 \pi \hbar} \int_{-\infty}^{\infty} d q\left[\left|1-r_{q}^{*}(-e V / 2) r_{q}(\hbar \Omega-e V / 2)\right|^{2}\right. \\
& \left.-\left|1-r_{q}^{*}(-e V / 2-\hbar \Omega) r_{q}(-e V / 2)\right|^{2}\right],
\end{aligned}
$$

$$
\begin{aligned}
\frac{d C_{R \rightarrow R}}{d V}= & \frac{e^{2} \Theta(\Omega)}{4 \pi \hbar} \int_{-\infty}^{\infty} d q\left[T_{q}(e V / 2) T_{q}(e V / 2+\hbar \Omega)\right. \\
& \left.-T_{q}(e V / 2-\hbar \Omega) T_{q}(e V / 2)\right], \\
\frac{d C_{L \rightarrow R}}{d V}= & \frac{e^{2} \Theta(\Omega-e V)}{4 \pi \hbar} \int_{-\infty}^{\infty} d q\left[T_{q}(\hbar \Omega-e V / 2)\right. \\
& \left.\times R_{q}(-e V / 2)-T_{q}(e V / 2) R_{q}(e V / 2-\hbar \Omega)\right],
\end{aligned}
$$

$$
\begin{aligned}
\frac{d C_{R \rightarrow L}}{d V}= & \frac{e^{2} \Theta(\Omega+e V)}{2 \pi \hbar} \int_{-\infty}^{\infty} d q\left[T_{q}(e V / 2) R_{q}(e V / 2+\hbar \Omega)\right. \\
& \left.-T_{q}(-e V / 2-\hbar \Omega) R_{q}(-e V / 2)\right] .
\end{aligned}
$$

Scattering amplitudes are roughly constant for a given $q$ mode in this regime, so the correlators are straight lines as a function of dc-bias voltage. For example, a special situation that could exhibit interesting physics is when some derivatives are zero. But this is, due to symmetry arguments, only possible at $e V=0, \pm \hbar \Omega$, proofing a zero slope of the correlators at their onsets but revealing no additional effect. By this means, as in the shot-noise spectrum, the dependence on the bias voltage reveals again the onsets of the four correlators. Since we have chosen positive $\hbar \Omega$, the autoterminal contributions are nonzero over the whole bias range. As before, cross-terminal ones vanish if no energy is provided by the voltage source: $C_{L \rightarrow R} \neq 0$ if $e V>-\hbar \Omega$ and $C_{R \rightarrow L} \neq 0$ if $e V<\hbar \Omega$. As it is clear from the bottom plot of Fig. 13, the oscillations of the components are not in phase, thus adding up to complicated oscillations in $S_{L L}(\Omega)$. Yet, as mentioned in the
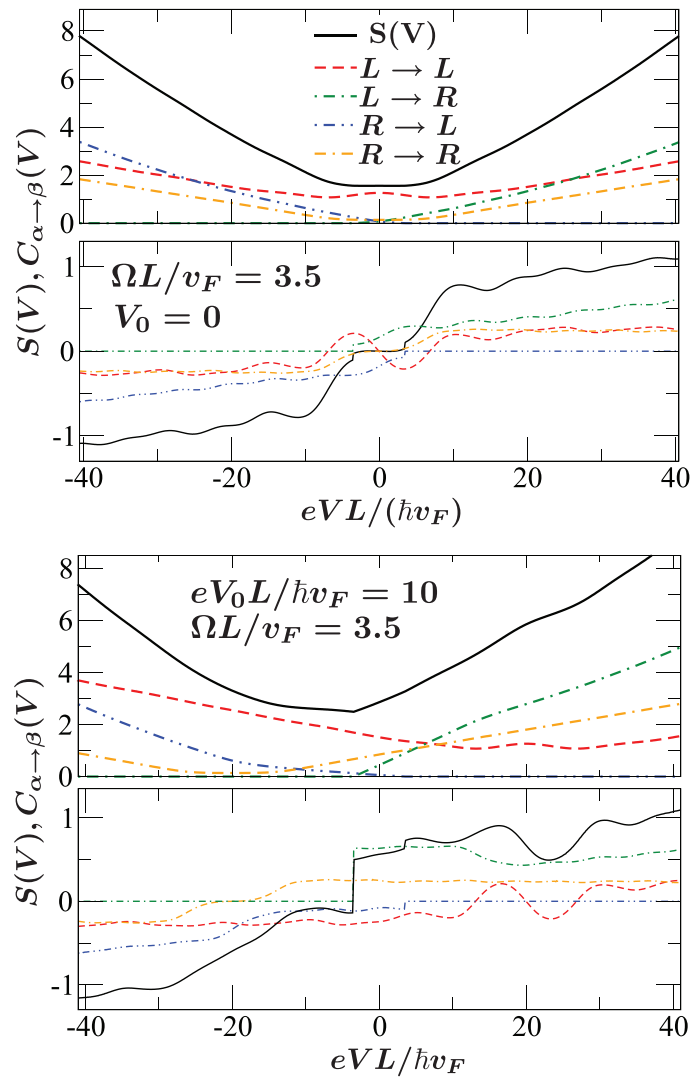

FIG. 13. (Color online) Real parts of autocorrelation currentcurrent fluctuations in units of $2 \pi \hbar / e^{2}$ as a function of dc bias for fixed frequency $\hbar \Omega$ (thick lines, top). We compare the symmetric setup without dc-bias offset (top) and when $e V_{0} L / \hbar v_{F}=10$ (bottom). Thin lines (lower panels) are used for the derivatives with respect to voltage. (Top) Due to symmetrically applied bias voltage, the noise and the autoterminal contributions are symmetric in the voltage dependence and $\left.S_{\alpha \rightarrow \beta}(\Omega)\right|_{V}=\left.S_{\beta \rightarrow \alpha}(\Omega)\right|_{-V}$ if $\alpha \neq \beta$. (Bottom) By applying an offset voltage, we are breaking the setup's symmetry. Autoterminal terms are then symmetric with respect to $e V= \pm 2 e V_{0}$, while the summed-up noise is asymmetric. 
beginning, we doubt this could be a measurable effect. The shot-noise and the autoterminal correlators are symmetric in the voltage dependence if $V_{0}=0$, whereas the cross-terminal ones obey $C_{\alpha \rightarrow \beta}(\Omega, V)=C_{\beta \rightarrow \alpha}(\Omega,-V)$. Here, the chargeneutrality point and the width of the region $\mathrm{III}_{b}$ are revealed as a minima in the slope of the correlator $C_{L \rightarrow L}(\Omega)$ at $e V= \pm 2 \hbar \Omega$ and in the change of sign in $d C_{R \rightarrow R}(\Omega) / d V$ at $e V=0$.

\section{CROSS-CORRELATION NOISE}

The explicit expressions of Eq. (27) for the cross-correlation current noise spectrum of Fig. 14 can be extracted from the general expression (21) in the same way as we did when deriving Eq. (24). From Figs. 8(a) and 8(b), it is also clear that the spectra of autoterminal correlators are oscillating as a function of $\Omega$ with larger amplitude than the cross-terminal ones, since they show an alternating behavior between positive and negative integrands. The dependence on $q$ in the relevant frequency range is weak, as shown in Fig. 8(a). Contrary, cross-terminal contributions as in Fig. 8(b) show features with an alternating sign along both variables, $\Omega$ and $q$. Thus an integration along $y$ momentum leads to averaging and therefore significantly smaller oscillation amplitudes occur. As discussed for the excess noise of the autocorrelation
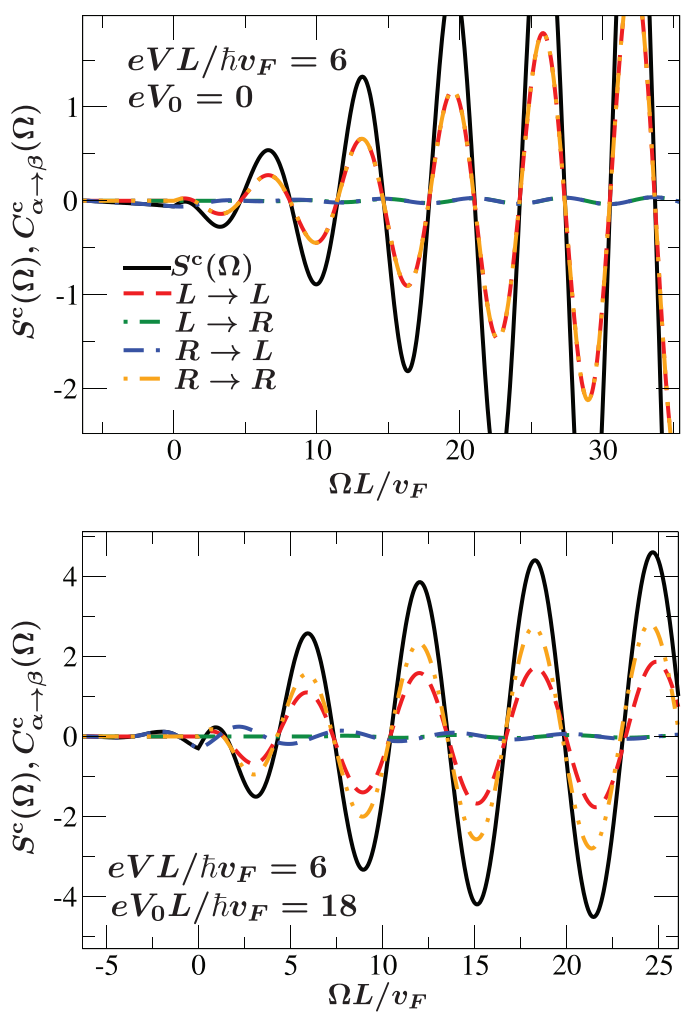

FIG. 14. (Color online) Real parts of cross-correlation spectrum in units of $2 \pi \hbar / e^{2}$ when $e V_{0}=0$ (top) and $e V_{0} L / \hbar v_{F}=$ $3 e V L / \hbar v_{F}=18$ (bottom). Without an offset voltage $\left(e V_{0}=0\right)$, autoterminal contributions are identical as well as cross-terminal ones at large frequencies $\hbar \Omega \gg e V$. At finite $e V_{0}$, the asymmetric bias voltage is reflected in the frequency dependence of the autoterminal correlators by their different heights and the shift of the oscillations maxima.

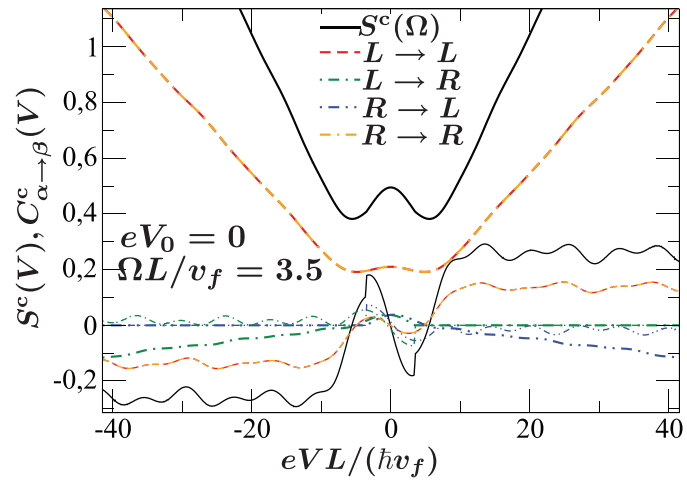

FIG. 15. (Color online) Real parts of current-current crosscorrelations in units of $2 \pi \hbar / e^{2}$ as a function of symmetrically applied dc-bias voltage and for a fixed frequency. Jumps in the derivatives at $\pm \Omega L / v_{F}$ are due to the onsets of the cross-terminal contributions.

noise spectral function, we find that the oscillations have a frequency $\hbar \Omega_{Z}=2 e V$, which is tantamount to a period $2 \pi$ in the plots. Complex conjugation corresponds to time-reversed states. Again, as the product of scattering matrices of the integrands in Eq. (21) suggests, it is probing transmission and reflection amplitudes of electron-hole pairs separated by an energy quanta $\hbar \Omega$. So, for cross-terminal noise, not only the reflection but also the complex transmission amplitude is essential even without ac-bias voltages. Again, it would be interesting to test if the resulting oscillations could be detected in the challenging task of a finite-frequency crosscorrelations experiment. Analyzing the integrands reveals the symmetry $C_{\alpha \rightarrow \alpha}^{\mathrm{c}}(\Omega)=C_{\beta \rightarrow \beta}^{\mathrm{c}}(\Omega)$ if $\mu_{L}=-\mu_{R}$ as we show in Fig. 15. This symmetry is distorted by applying an offset voltage $V_{0}$. The spectrum of the correlator $C_{L \rightarrow L}^{\mathrm{c}}(\Omega)$ shows a shift of the maxima and minima of the oscillations with respect to $C_{R \rightarrow R}^{\mathrm{c}}(\Omega)$ for finite $V_{0}$. This shift is due to the fact that the distance between neighboring maxima of the integrand is not constant when varying $\hbar \Omega$ at given $q$ mode (see the bending of the maxima towards higher frequencies for larger $q$ in the integrands, e.g., Fig. 6). Derivatives of the correlators $C_{\alpha \rightarrow \beta}^{\mathrm{c}}(\Omega)$ with respect to voltage show a sequence of pairs of different maxima. This observation is traced down to the same origin as above, and so the appearance of peculiar oscillations in the summed-up crosscorrelation shot noise $S_{L R}(\Omega)$ is explained. At $\hbar \Omega=0$, current conservation and the unitarity of the $s$ matrix require $S_{L R}(\Omega)=$ $-S_{L L}(\Omega)$. Therefore the correlator described by Eq. $(27 \mathrm{~d})$ is negative.

\section{FINITE-FREQUENCY NOISE AT AC BIAS}

By applying an ac-bias voltage at the leads, one can inject charge carriers at positive and negative energies of the Dirac cone without applying a dc voltage. Analogous to the minimal conductivity, in the nondriven case going along with a maximal Fano factor, the shot noise at zero frequency but finite ac bias $S_{\alpha \alpha}(\Omega=0 ; \omega)$ mirrors the behavior of the conductivity in Fig. 3. The noise spectrum in Fig. 16 for the driven setup $(a=1)$ is similar to the one without driving but with additional steps in the derivatives. For arbitrary ac bias, these steps can appear at frequencies $\hbar \Omega=\left(\mu_{\alpha}-\mu_{\beta}\right) \pm n \hbar \omega$ due to the onset 


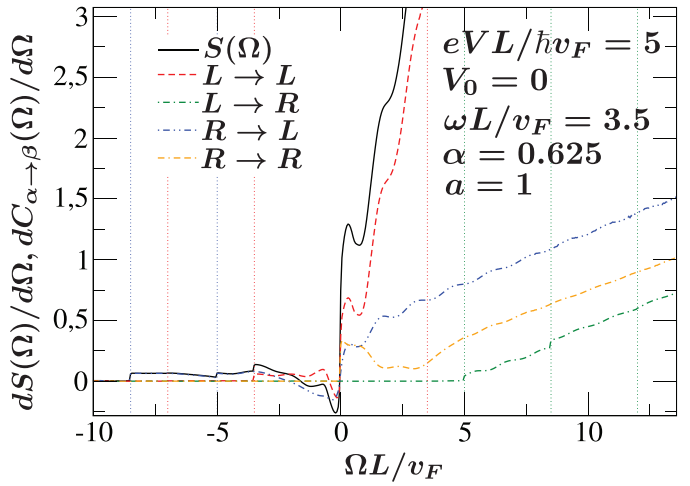

FIG. 16. (Color online) Derivatives of current-current correlations real parts in units of $2 \pi \hbar / e^{2}$ with respect to frequency as a function of frequency. We have chosen a symmetrically applied dc-bias voltage with additional harmonic ac driving $\left(\omega L / v_{F}=3.5\right.$, $\alpha=0.5$ and $a=1$ ) in lead $L$. Dashed vertical lines mark the step positions, coloring specifies the correlator that shows the step at corresponding $\Omega L / v_{F}$.

of higher-order PAT events. Since we set $a=1$ in Fig. 16, the correlator with states $R \rightarrow R$ shows no ac-induced steps in the derivative. But when $|a| \neq 1$, all integrands (see Fig. 9) are not given in terms of probabilities and can take negative values as mentioned in Sec. V. For the shot-noise spectrum, there are then two possible sources of contributions that could reduce noise: either a correlators integrand or the product of Bessel functions is negative. When the driving voltage is applied symmetrically $(a=0)$, more PAT-induced steps in the derivatives of the noise spectrum are visible and finite contributions at negative $\Omega$ are possible for all correlators. As proposed by Trauzettel et al., ${ }^{43}$ a time-dependent voltage could be used to induce interference between states in particle- and holelike parts of the Dirac spectrum. This should correspond to Zitterbewegung like in relativistic quantum mechanics, but we are not aware of any unique feature caused by Zitterbewegung that can be distinguished from other oscillations, especially of Fabry-Pérot nature.

\section{CONCLUSIONS}

We have analyzed the conductivity and the nonsymmetrized finite-frequency current-current correlations for a Fabry-Pérot graphene structure. Oscillations on the intrinsic energy scale $L / \hbar v_{F}$ are still present in the finite frequency noise. Emission spectra are diverging for large frequencies, whereas the absorption branch of the spectrum has to vanish at $\hbar \Omega=-e V$. As expected from the integrands, the current noise also diverges for voltages $|e V| \gg \hbar v_{F} / L$. Since the onset of the different noise contributions is defined by the four possible combinations of the chemical potentials, the noise built by all correlators consists of contributions oscillating with the same period but different phases. Although dominated by $C_{L \rightarrow L}(\Omega)$ when correlating the currents in terminal $L$ at large frequencies, this interplay is revealed in the spectra and voltage dependence of all correlators. Each contribution can show peculiar oscillations at low enough frequencies or voltages. In this regime, features in the integrands $(q, \epsilon)$ dependence can have a prominent impact whereas they tend to be averaged out at large frequencies. Another aspect is the appearance of a special region showing phase jumps in the energy dependence of the integrands when $\hbar \Omega \leqslant 2 e V$. This interplay of the Dirac spectrum and the Fabry-Pérot physics ${ }^{79,80}$ can be probed purely by applying an appropriate combination of dc-bias and offset voltage $V_{0}=V / 2$, thus connecting electron and hole parts of the Dirac spectrum symmetrically when $e V=2 \hbar \Omega$. The way the scattering amplitudes are combined in this approach destroys the clear picture in terms of transmission and reflection probabilities. Instead, in the dc limit, it gives rise to the interpretation of the $L \rightarrow L$ contribution in terms of jumps in the scattering phase between time-reversed electronhole states separated by the photon energy $\hbar \Omega$. In the same way, the complex correlators for cross-correlation noise or for the driven setup exhibit phase jumps and can not be written in terms of probabilities. Complex contributions of the scattering matrices lead to large oscillations between positive and negative values of cross-correlation noise or in the derivatives with respect to frequency of the autoterminal noise spectral function. These oscillations have a frequency of $\hbar \Omega_{Z}=2 e V$, which corresponds to a period of $2 \pi$ in our plots. This frequency corresponds to the Zitterbewegung frequency as it is known for relativistic Dirac fermions. Again, strongly nonharmonic features can occur when the transition between different regimes is probed, especially when region $\mathrm{III}_{b}$ around the Dirac point comes into play. Additional ac bias complicates the picture because combinations of $q, \hbar \Omega, m \hbar \omega$ define additional phase jumps, onsets of the correlators and therefore steps in the noise when higher-order PAT events occur. Then the special role of the complex reflection and transmission amplitudes is essential for all possible correlators. These complex reflection and transmission amplitudes are a central and common feature of frequency-dependent shot noise in double-barrier structures, which we studied recently for a single resonant level ${ }^{48}$ using the same approach as in this work. In this simple system, the onsets of the correlators are the same as in the graphene setup since they only depend on the considered voltages and frequencies. Apart from this aspect, the frequency dependence of the single resonant level and that for the graphene system differ strongly. Most of all, the oscillatory behavior of the shot noise spectra in the graphene setup cannot be found in the case of the resonant level that rather leads to a steplike structure of the frequency-dependent shot noise.

Thus it seem worth to investigate a graphene Fabry-Pérot setup experimentally as the frequency scales are in the accessible regime and the noise correlators can (partially) reveal the physics of the Zitterbewegung. Future theoretical studies might investigate the screening effects on the noise properties in the very high-frequency regime, which gives information about the relevance of displacement currents in the relativistic regime.

\section{ACKNOWLEDGMENTS}

We would like to acknowledge the financial support by the DFG and SFB 767 and thank B. Trauzettel for validating and sharing corrections to Ref. 43. 


\section{APPENDIX A: NOISE FORMULAS}

The nonsymmetrized noise spectrum under harmonic ac driving without interactions is determined by

$$
\begin{aligned}
S_{\alpha \beta}(\Omega, \omega)= & \left(\frac{e^{2}}{2 \pi \hbar}\right) \int d \epsilon \sum_{\gamma \delta, l k m} J_{l}\left(\frac{e V_{\mathrm{ac}, \gamma}}{\hbar \omega}\right) J_{k}\left(\frac{e V_{\mathrm{ac}, \delta}}{\hbar \omega}\right) \\
& \times J_{m+k-l}\left(\frac{e V_{\mathrm{ac}, \delta}}{\hbar \omega}\right) J_{m}\left(\frac{e V_{\mathrm{ac}, \gamma}}{\hbar \omega}\right) \\
& \times \operatorname{Tr}\left[A_{\gamma \delta}(\alpha, \epsilon, \epsilon+\hbar \Omega) A_{q ; \delta \gamma}(\beta, \epsilon+\hbar \Omega\right. \\
& +(m-l) \hbar \omega, \epsilon+(m-l) \hbar \omega)] f_{\gamma}(\epsilon-l \hbar \omega) \\
& \times\left[1-f_{\delta}(\epsilon+\hbar \Omega-k \hbar \omega)\right] .
\end{aligned}
$$

For the Fermi distribution function in lead $\gamma$, we use the shorthand $f_{\gamma}(\epsilon)=1 /\left\{\exp \left[\left(\epsilon-\mu_{\gamma}\right) / k_{B} T\right]+1\right\}$. In the limit of $k_{B} T=0$, the distribution functions in the leads are given by Heaviside $\Theta$ functions $\Theta\left(\mu_{\gamma}-\epsilon\right)$ that define the integration intervals. Explicitly writing down the expression above for chosen $\alpha, \beta$ then leads to the four possible contributions to the auto-correlation noise of Eq. (24) and to the cross-correlation noise of Eq. (27) via summation over reservoir indices $\gamma, \delta=$ $L, R$.

\section{APPENDIX B: BOUNDARY CONDITIONS}

As in Ref. 20, we confine the charge carriers along the $y$ directions by infinite mass boundaries that diverge at the edges $y=0, W$. This corresponds to the boundary conditions $^{47}$

$$
\left.\hat{\Psi}_{1}\right|_{y=0}=\left.\left.\hat{\Psi}_{2}\right|_{y=0} \quad \hat{\Psi}_{1}\right|_{y=W}=-\left.\hat{\Psi}_{2}\right|_{y=W} .
$$

Unlike the procedure for the Schrödinger equation, in graphene one only has to match the wave function itself and no constraint is given for the derivatives. Now exploiting the boundary conditions along the $x$ direction, transmission and reflection amplitudes are fully determined by these constraints of the field operators at the Fermi levels. Hence it is sufficient to match the wave functions at $x=0, L$ without ac driving. A plain wave ansatz to solve the Dirac equation (1) for an electron incident from the left $(x<0)$ with energy $\epsilon$ is given by

$$
\Psi(\mathbf{x})=\left\{\begin{array}{lll}
\Psi_{0,+}^{\kappa_{q}^{L}(\epsilon), q}+r_{q}(\epsilon) \Psi_{0,-}^{\kappa_{q}^{L}(\epsilon), q}, & \text { if } \quad x<0, \\
a_{q}(\epsilon) \Psi_{0,+}^{k_{q}(\epsilon), q}+b_{q}(\epsilon) \Psi_{0,-}^{k_{q}(\epsilon), q}, & \text { if } \quad 0<x<L, \\
t_{q}(\epsilon) \Psi_{0,+}^{\kappa_{q}^{R}(\epsilon), q} e^{-i \kappa_{q}^{R}(\epsilon) L}, & \text { if } \quad x>L,
\end{array}\right.
$$

where $\kappa_{q}^{L / R}(\epsilon)=\frac{\epsilon+\mu_{L / R}}{\hbar v_{F}} \cos \left[\alpha_{q}(\epsilon)\right]$ are the wave vectors in the reservoirs and $k_{q}(\epsilon)$ is the one in the graphene strip. Matching conditions at boundaries (continuity at $x=0, L$ ) combined with high doping in the reservoirs lead to the following set of coupled, complex equations:

$$
\begin{aligned}
& \frac{1+r_{q}(\epsilon)}{\sqrt{2}}=a_{q}(\epsilon) \frac{e^{-i \alpha_{q}(\epsilon) / 2}}{\sqrt{\cos \left[\alpha_{q}(\epsilon)\right]}}+b_{q}(\epsilon) \frac{e^{i \alpha_{q}(\epsilon)}}{\sqrt{\cos \left[\alpha_{q}(\epsilon)\right]}}, \\
& \frac{1-r_{q}(\epsilon)}{\sqrt{2}}=a_{q}(\epsilon) \frac{e^{i \alpha_{q}(\epsilon) / 2}}{\sqrt{\cos \left[\alpha_{q}(\epsilon)\right]}}-b_{q}(\epsilon) \frac{e^{-i \alpha_{q}(\epsilon)}}{\sqrt{\cos \left[\alpha_{q}(\epsilon)\right]}}, \\
& \frac{t_{q}(\epsilon)}{\sqrt{2}}=a_{q}(\epsilon) \frac{e^{i k_{q}(\epsilon) L} e^{-i \alpha_{q}(\epsilon) / 2}}{\sqrt{\cos \left[\alpha_{q}(\epsilon)\right]}}+b_{q}(\epsilon) \frac{e^{-i k_{q}(\epsilon) L} e^{i \alpha_{q}(\epsilon)}}{\sqrt{\cos \left[\alpha_{q}(\epsilon)\right]}}, \\
& \frac{t_{q}(\epsilon)}{\sqrt{2}}=a_{q}(\epsilon) \frac{e^{i k_{q}(\epsilon) L} e^{i \alpha_{q}(\epsilon) / 2}}{\sqrt{\cos \left[\alpha_{q}(\epsilon)\right]}}-b_{q}(\epsilon) \frac{e^{-i k_{q}(\epsilon) L} e^{-i \alpha_{q}(\epsilon)}}{\sqrt{\cos \left[\alpha_{q}(\epsilon)\right]}} .
\end{aligned}
$$

The solution is straightforward and determined by the four complex coefficients $a_{q}(\epsilon), b_{q}(\epsilon), t_{q}(\epsilon), r_{q}(\epsilon)$. The transmission $t_{q}(\epsilon)$ and reflection $r_{q}(\epsilon)$ amplitudes define the $s$ matrix and thus the current and noise of the scattering device at all voltages:

$$
\begin{aligned}
& t_{q}(\epsilon)=\frac{1}{\left.\cos \left[k_{q}(\epsilon) L\right)\right]-i \sec \left[\alpha_{q}(\epsilon)\right] \sin \left[k_{q}(\epsilon) L\right]} \\
& r_{q}(\epsilon)=\frac{1}{-\cot \left[k_{q}(\epsilon) L\right] \cot \left[\alpha_{q}(\epsilon)\right]+i \csc \left[\alpha_{q}(\epsilon)\right]}, \\
& a_{q}(\epsilon)=\frac{\sqrt{2} \cos \left[\frac{\alpha_{q}(\epsilon)}{2}\right] \sqrt{\cos \left[\alpha_{q}(\epsilon)\right]}}{1+e^{2 i k_{q}(\epsilon) L}\left\{-1+\cos \left[\alpha_{q}(\epsilon)\right]\right\}+\cos \left[\alpha_{q}(\epsilon)\right]}, \\
& b_{q}(\epsilon)=-\frac{e^{i k_{q}(\epsilon) L} \sqrt{\cos \left[\alpha_{q}(\epsilon)\right]} \sin \left[\frac{\alpha_{q}(\epsilon)}{2}\right]}{\sqrt{2} \backslash\left\{i \cos \left[k_{q}(\epsilon) L\right] \cos \left[\alpha_{q}(\epsilon)\right]+\sin \left[k_{q}(\epsilon) L\right]\right\}}
\end{aligned}
$$

${ }^{9}$ E. B. Sonin, Phys. Rev. B 79, 195438 (2009)

${ }^{10}$ A. F. Young and Philip Kim, Nat. Phys. 5, 222 (2009).

${ }^{11}$ A. V. Shytov, M. S. Rudner, and L. S. Levitov, Phys. Rev. Lett. 101, 156804 (2008).

${ }^{12}$ M. I. Katsnelson, Eur. Phys. J. B 51, 157 (2006).

${ }^{13}$ J. Schliemann, Phys. Rev. B 77, 125303 (2008).

${ }^{14}$ T. M. Rusin and W. Zawadzki, Phys. Rev. B 76, 195439 (2007).

${ }^{15}$ M. I. Katsnelson, Eur. Phys. J. B 52, 151 (2006).

${ }^{16}$ K. Ziegler, Phys. Rev. B 75, 233407 (2007).

${ }^{17}$ I. Snyman, J. Tworzydło, and C. W. J. Beenakker, Phys. Rev. B 78, 045118 (2008).

${ }^{18}$ K. S. Novoselov, A. K. Geim, S. V. Morozov, D. Jiang, M. I. Katsnelson, I. V. Grigorieva, S. V. Dubonos, and A. A. Firsov, Nature (London) 438, 197 (2005). 
${ }^{19}$ Y. Zhang, Y.-W. Tan, H. L. Stormer, and P. Kim, Nature (London) 438, 201 (2005).

${ }^{20}$ J. Tworzydło, B. Trauzettel, M. Titov, A. Rycerz, and C. W. J. Beenakker, Phys. Rev. Lett. 96, 246802 (2006).

${ }^{21}$ R. Zhu and Y. Guo, Appl. Phys. Lett. 91, 252113 (2007).

${ }^{22}$ L. DiCarlo, J. R. Williams, Y. Zhang, D. T. McClure, and C. M. Marcus, Phys. Rev. Lett. 100, 156801 (2008).

${ }^{23}$ C. W. Groth, J. Tworzydło, and C. W. J. Beenakker, Phys. Rev. Lett. 100, 176804 (2008).

${ }^{24}$ R. Danneau, F. Wu, M. F. Craciun, S. Russo, M. Y. Tomi, J. Salmilehto, A. F. Morpurgo, and P. J. Hakonen, Phys. Rev. Lett. 100, 196802 (2008).

${ }^{25}$ C. W. J. Beenakker and M. Büttiker, Phys. Rev. B 46, 1889 (1992).

${ }^{26}$ K. E. Nagaev, Phys. Lett. A 169, 103 (1992).

${ }^{27}$ C. H. Lewenkopf, E. R. Mucciolo, and A. H. Castro Neto, Phys. Rev. B 77, 081410 (2008).

${ }^{28}$ A. Rycerz, P. Recher, and M. Wimmer, Phys. Rev. B 80, 125417 (2009).

${ }^{29}$ E. B. Sonin, Phys. Rev. B 77, 233408 (2008).

${ }^{30}$ V. P. Gusynin, S. G. Sharapov, and J. P. Carbotte, Phys. Rev. Lett. 96, 256802 (2006).

${ }^{31}$ F. J. Lopez-Rodriguez and G. G. Naumis, Phys. Rev. B 78, 201406 (2008).

${ }^{32}$ M. Ramezani Masir, P. Vasilopoulos, and F. M. Peeters, Phys. Rev. B 82, 115417 (2010).

${ }^{33}$ S. Gattenlöhner, W. Belzig, and M. Titov, Phys. Rev. B 82, 155417 (2010).

${ }^{34}$ J. R. Williams, L. DiCarlo, and C. M. Marcus, Science 317, 638 (2007).

${ }^{35}$ D. A. Abanin and L. S. Levitov, Science 317, 5838 (2007).

${ }^{36}$ M. V. Fistul and K. B. Efetov, Phys. Rev. Lett. 98, 256803 (2007).

${ }^{37}$ S. V. Syzranov, M. V. Fistul, and K. B. Efetov, Phys. Rev. B 78, 045407 (2008).

${ }^{38}$ Ya. Blanter and M. Büttiker, Phys. Rep. 336, 1 (2000).

${ }^{39}$ P. K. Tien and J. P. Gordon, Phys. Rev. 129, 647 (1962).

${ }^{40}$ M. Grifoni and P. Hänggi, Phys. Rep. 304, 229 (1998).

${ }^{41}$ G. Platero and R. Aguado, Phys. Rep. 395, 1 (2004).

${ }^{42}$ S. Kohler, J. Lehmann, and P. Hänggi, Phys. Rep. 406, 379 (2005)

${ }^{43}$ B. Trauzettel, Ya. M. Blanter, and A. F. Morpurgo, Phys. Rev. B 75, 035305 (2007).

${ }^{44}$ C. W. J. Beenakker, Phys. Rev. Lett. 97, 067007 (2006).

${ }^{45}$ C. G. Rocha, Luis E. F. Foa Torres, and G. Cuniberti, Phys. Rev. B 81, 115435 (2010).

${ }^{46}$ M. A. Zeb, K. Sabeeh, and M. Tahir, Phys. Rev. B 78, 165420 (2008).

${ }^{47}$ M. V. Berry and R. J. Mondragon, Proc. R. Soc. London A 412, 53 (1987).

${ }^{48}$ J. Hammer and W. Belzig, Phys. Rev. B 84, 085419 (2011).

${ }^{49}$ F. Miao, S. Wijeratne, Y. Zhang, U. C. Coskun, W. Bao, and C. N. Lau, Science 317, 1530 (2007).

${ }^{50}$ M. A. Laakso and T. T. Heikkila, Phys. Rev. B 78, 205420 (2008).

${ }^{51}$ A. Schuessler, P. M. Ostrovsky, I. V. Gornyi, and A. D. Mirlin, Phys. Rev. B 79, 075405 (2009).

${ }^{52}$ M. I. Katsnelson and F. Guinea, Phys. Rev. B 78, 075417 (2008).
${ }^{53}$ D. C. Guhr, D. Rettinger, J. Boneberg, A. Erbe, P. Leiderer, and E. Scheer, Phys. Rev. Lett. 99, 086801 (2007).

${ }^{54}$ M. H. Pedersen and M. Büttiker, Phys. Rev. B 58, 12993 (1998).

${ }^{55}$ M. Büttiker, A. Prêtre, and H. Thomas, Phys. Rev. Lett. 70, 4114 (1993).

${ }^{56}$ N. M. R. Peres, A. H. Castro Neto, and F. Guinea, Phys. Rev. B 73, 195411 (2006).

${ }^{57}$ M. Titov, Europhys. Lett. 79, 17004 (2007).

${ }^{58}$ R. J. Schoelkopf, P. J. Burke, A. A. Kozhevnikov, D. E. Prober, and M. J. Rooks, Phys. Rev. Lett. 78, 3370 (1997).

${ }^{59}$ G. Lesovik and R. Loosen, JETP Lett. 65, 295 (1997).

${ }^{60}$ U. Gavish, Y. Levinson, and Y. Imry, Phys. Rev. B 62, 10637 (2000).

${ }^{61}$ U. Gavish, Y. Levinson, and Y. Imry, Proceedings of the 2001 Rencontres de Moriond: Electronic Correlations: from Meso- to Nanophysics, edited by T. Martin, G. Montambaux, and J. Tran Thanh Van (EDP Science, Les Ulis, 2001).

${ }^{62}$ C. W. J. Beenakker and H. Schomerus, Phys. Rev. Lett. 86, 700 (2001).

${ }^{63}$ L. H. Reydellet, P. Roche, D. C. Glattli, B. Etienne, and Y. Jin, Phys. Rev. Lett. 90, 176803 (2003).

${ }^{64}$ B. Reulet, J. Senzier, and D. E. Prober, Phys. Rev. Lett. 91, 196601 (2003).

${ }^{65}$ Quantum Noise in Mesoscopic Physics, edited by Y. V. Nazarov (Kluwer, Dordrecht, 2008).

${ }^{66}$ J. Gabelli and B. Reulet, Phys. Rev. Lett. 100, 026601 (2008).

${ }^{67}$ J. Gabelli and B. Reulet, J. Stat. Mech. (2009) P01049.

${ }^{68}$ E. Zakka-Bajjani, J. Ségala, F. Portier, P. Roche, D. C. Glattli, A. Cavanna, and Y. Jin, Phys. Rev. Lett. 99, 236803 (2007).

${ }^{69}$ H.-P. Engel and D. Loss, Phys. Rev. Lett. 93, 136602 (2004).

${ }^{70}$ O. Entin-Wohlman, Y. Imry, S. A. Gurvitz, and A. Aharony, Phys. Rev. B 75, 193308 (2007).

${ }^{71}$ E. A. Rothstein, O. Entin-Wohlman, and A. Aharony, Phys. Rev. B 79, 075307 (2009).

${ }^{72}$ C. Schönenberger and C. Beenakker, Phys. Today 56, 37 (2003).

${ }^{73}$ R. Aguado and L. P. Kouwenhoven, Phys. Rev. Lett. 84, 1986 (2000).

${ }^{74}$ R. Aguado and T. Brandes, Phys. Rev. Lett. 92, 206601 (2004).

${ }^{75}$ D. Marcos, C. Emary, T. Brandes, and R. Aguado, Phys. Rev. B 83, 125426 (2011)

${ }^{76}$ M. Büttiker, Phys. Rev. B 45, 3807 (1992).

${ }^{77}$ M. Büttiker, H. Thomas, and A. Prêtre, Z. Phys. B 94, 133 (1994).

${ }^{78} \mathrm{M}$. Büttiker and T. Christen, in Quantum Transport in Semiconductor Submicron Structures, edited by B. Kramer (Kluwer, Dordrecht, 1996).

${ }^{79}$ W. Liang, M. Bockrath, D. Bozovic, J. H. Hafner, M. Tinkham, and H. Park, Nature (London) 411, 665 (2001).

${ }^{80}$ L. G. Herrmann, T. Delattre, P. Morfin, J.-M. Berroir, B. Plaçais, D. C. Glattli, and T. Kontos, Phys. Rev. Lett. 99, 156804 (2007).

${ }^{81}$ M. Creux, A. Crepieux, and T. Martin, Phys. Rev. B 74, 115323 (2006).

${ }^{82}$ A. V. Lebedev, A. Crépieux, and T. Martin, Phys. Rev. B 71, 075416 (2005).

${ }^{83}$ S. Oberholzer, E. V. Sukhorukov, and C. Schönenberger, Nature (London) 415, 765 (2002). 